\title{
Polyphasic Taxonomy in the Genus Burkholderia Leading to an Emended Description of the Genus and Proposition of Burkholderia vietnamiensis sp. nov. for $\mathrm{N}_{2}$-Fixing Isolates from Rice in Vietnam
}

\author{
MONIQUE GILLIS, ${ }^{1 *}$ TRAN VAN VAN,${ }^{2}$ RENÉ BARDIN,${ }^{3}$ MART GOOR, ${ }^{1}$ PRAKASH HEBBAR, ${ }^{2}$ \\ ANNE WILLEMS, ${ }^{1}$ PAUL SEGERS, ${ }^{1}$ KAREL KERSTERS, ${ }^{1}$ THIERRY HEULIN, ${ }^{2}$ AND MARIA P. FERNANDEZ ${ }^{3}$ \\ Laboratorium voor Microbiologie, Universiteit Gent, B-9000 Ghent, Belgium, ${ }^{1}$ and Equipe d'Ecologie Microbienne \\ de la Rhizosphère, Centre de Pédologie Biologique, UPR 6831 du CNRS, associée à l'Université de Nancy I, \\ F-54501 Vandouvre-les-Nancy, ${ }^{2}$ and URA CNRS 1977, Ecologie Microbienne, LASBSE,
}

F-69622 Villeurbanne Cédex, ${ }^{3}$ France

\begin{abstract}
The taxonomic position of nitrogen-fixing strains that were isolated from rhizosphere macerates of rice cultivated in the Bình Thanh region of Vietnam was determined by using polyphasic taxonomy. We determined the phylogenetic relationships of these organisms by performing DNA-rRNA hybridization experiments with a labeled rRNA probe from the type strain of Burkholderia cepacia, and we found that they belong to a single rRNA complex. Other members of this rRNA complex were also studied, and the $\mathbf{N}_{2}$-fixing strains were found to be closely related to $B$. cepacia. In addition, all members of the rRNA complex containing $B$. cepacia were studied by performing auxanographic and DNA-DNA hybridization experiments. Phenotypically and genotypically, the $\mathbf{N}_{\mathbf{2}}$-fixing isolates constitute a single cluster together with two strains of clinical origin. These organisms constitute a new Burkholderia species, for which the name Burkholderia vietnamiensis is proposed; the type strain of this species is TVV75 (= LMG 10929). All members of this species can fix nitrogen. On the basis of our polyphasic taxonomy results and previously published data we concluded that the genus Burkholderia should be restricted to the following species: $B$. cepacia (the type species), Burkholderia mallei, Burkholderia pseudomallei, B. vietnamiensis, Burkholderia gladioli, Burkholderia caryophylli, Burkholderia plantarii, Burkholderia glumae, Burkholderia vandii, Burkholderia cocovenenans comb. nov., and Burkholderia andropogonis comb. nov. On the basis of genotypic and phenotypic results [Alcaligenes] eutrophus, [Burkholderia] solanacearum, and [Burkholderia] pickettii belong to two other clusters whose internal structures must be studied further.
\end{abstract}

On the basis of the results of extensive phenotypic studies and DNA-rRNA and DNA-DNA hybridization experiments performed by Palleroni, Stanier, and their collaborators, the genus Pseudomonas was divided into five groups $(24,43,46$, 56). Additional phylogenetic data have shown that these groups are only very remotely related and that each of them contains species belonging to other genera $(21-23,70,71)$. In addition to the former pseudomonads that have been shown to belong to the genus Xanthomonas and to a smaller group related to Pseudomonas diminuta and Pseudomonas vesicularis $(21-23,43)$ classified in the recently described genus Brevundimonas (52), three large groups of pseudomonads can be considered. Pseudomonas rRNA group I (43) is part of rRNA superfamily II (18) or the gamma subclass of the Proteobacteria (55), where it constitutes a separate rRNA complex $(18,21,22$, 68 ); this group represents the authentic pseudomonads which are grouped with Pseudomonas aeruginosa, the type species (43). Although the internal relationships within this group have not been determined yet, it is evident that the genus Pseudomonas must be limited to this group and that all other Pseudomonas species have been generically misnamed as determined by phylogenetic data. Below the genus names of misnamed taxa are enclosed in brackets. [Pseudomonas] rRNA group III (43) is part of the acidovorans rRNA complex in rRNA superfamily III or the beta subclass of the Proteobacteria. A detailed

* Corresponding author. Mailing address: Laboratorium voor $\mathrm{Mi}-$ crobiologie, K.-L. Ledeganckstraat, 35, B-9000 Ghent, Belgium. Phone: 329264 5117. Fax: 329264 5346. Electronic mail address: Moniek.Gillis@rug.ac.be. polyphasic approach has revealed the finer relationships within the acidovorans rRNA complex, and workers have created a new bacterial family, the Comamonadaceae (67), which contains in addition to the genus Comamonas several newly described genera, including the genera Hydrogenophaga, Acidovorax, Variovorax, and Xylophilus.

[Pseudomonas] rRNA group II (43) is also part of rRNA superfamily III, where it constitutes a separate cluster that is only remotely related to the Comamonadaceae (18); [Pseudomonas] rRNA group II and the Comamonadaceae belong to separate large rRNA branches that split off at $T_{m(e)}$ values of about $70^{\circ} \mathrm{C}\left[T_{m(e)}\right.$ is the temperature at which $50 \%$ of a DNArRNA duplex is denatured]. This rRNA cluster contains at least 10 [Pseudomonas] species, including several phytopathogenic species, as well as nonphytopathogenic species. These species are [Pseudomonas] cepacia, [Pseudomonas] solanacearum, [Pseudomonas] gladioli, [Pseudomonas] caryophylli, [Pseudomonas] glumae, [Pseudomonas] andropogonis, [Pseudomonas] woodsii, [Pseudomonas] pickettii, [Pseudomonas] phenazinium, and [Pseudomonas] oxalaticus (18). When rRNA from $\left[P\right.$. ] solanacearum NCPPB $325^{\mathrm{T}}(\mathrm{T}=$ type strain $)$ is used to form DNA-rRNA duplexes, these organisms have $T_{m(e)}$ values ranging from 74 to $81.5^{\circ} \mathrm{C}$. [Pseudomonas] mallei and [Pseudomonas] pseudomallei also belong to this group (43). Four other species ([Pseudomonas] plantarii [2], [Pseudomonas] mixta [8], [Pseudomonas] syzygii [50], and [Pseudomonas] cocovenenans [74]) have also been described as members of the so-called solanacearum rRNA group (68). Recently, Bowman et al. (9) provided evidence that [Pseudomonas] mixta belongs to another lineage in the beta subclass, and consequently these 
authors transferred this species to the new genus Telluria as Telluria mixta. Two generically misnamed Alcaligenes species, [Alcaligenes] eutrophus and "[Alcaligenes] hydrogenophilus," definitely belong to this rRNA complex (18). It has also been shown that $[P$.] oxalaticus is phenotypically and genotypically closely related to $[A$.] eutrophus (32). [Pseudomonas] rubrisubalbicans has also been described as a member of this rRNA group (23), but recently this placement has been refuted since this species constitutes a separate rRNA cluster that splits off at a $T_{m(e)}$ value of $73^{\circ} \mathrm{C}$ from the solanacearum rRNA branch $(18,25)$. The broad range of guanine-plus-cytosine $(\mathrm{G}+\mathrm{C})$ contents, the relatively broad range of $T_{m(e)}$ values, and the variations in phenotypic characteristics (3) among the species suggest that there is a certain amount of heterogeneity within the solanacearum rRNA complex. An analysis of the sequences of the 16S rRNA genes of representative strains of seven species by $\mathrm{Li}$ et al. (38) revealed that there are two lineages that correspond to one genus or possibly two related genera. Yabuuchi et al. (73) transferred seven species that belong to this rRNA complex to the new genus Burkholderia on the basis of the results of a polyphasic taxonomic study of the type or reference strains of the following species: Burkholderia mallei, Burkholderia pseudomallei, Burkholderia cepacia, Burkholderia solanacearum, Burkholderia caryophylli, Burkholderia gladioli, and Burkholderia pickettii. Later, Urakami et al. (63) also transferred $[P$.$] plantarii and [P$.$] glumae to the genus$ Burkholderia as Burkholderia plantarii and Burkholderia glumae, respectively, and created a new species, Burkholderia van$d i i$, for a strain isolated from the roots of Vanda species.

Recently, Tran Van (62) isolated several $\mathrm{N}_{2}$-fixing bacterial strains that are associated with roots of rice growing in rice fields in Vietnam. These strains have been further characterized, and it has been shown that they are phenotypically very similar to B. cepacia. The aims of this study were to further determine the internal structure of the solanacearum rRNA complex and to determine the relationship between the $\mathrm{N}_{2}$ fixing isolates and this group (particularly $B$. cepacia). In this study we used a polyphasic approach which included DNArRNA hybridization, DNA-DNA hybridization, and auxanographic analysis of as many strains of each species as possible. Our results clearly demonstrated that $B$. cepacia, $B$. gladioli, $B$. plantarii, $B$. glumae, $\left[P\right.$.] cocovenenans, and the $\mathrm{N}_{2}$-fixing isolates constitute a separate rRNA branch within the rRNA complex. Genotypically, the $\mathrm{N}_{2}$-fixing isolates are closely related to $B$. cepacia. We propose that the genus Burkholderia should be restricted to the members of this rRNA branch plus B. caryophylli, B. mallei, B. pseudomallei, B. vandii, and Burkholderia andropogonis comb. nov. and that a new species, Burkholderia vietnamiensis, should be created for the $\mathrm{N}_{2}$-fixing taxon. The former $[P$.] cocovenenans also belongs in the genus Burkholderia as Burkholderia cocovenenans comb. nov. [Alcaligenes] eutrophus and $[P$.$] oxalaticus constitute another rRNA$ branch, as do [Burkholderia] solanacearum and [Burkholderia] pickettii, and the finer relationships of these two branches have to be determined. These organisms represent at least one separate genus. Below we use the new nomenclature described above.

\section{MATERIALS AND METHODS}

Bacterial strains. The strains that we used are listed in Table 1. Most of these strains are culture collection strains; the exceptions are the TVV strains (TVV69, TVV70, TVV71, TVV72, TVV74, TVV75 ${ }^{\mathrm{T}}$, TVV115, TVV116, TVV127, TVV128, TVV131, and TVV135), which were isolated from rhizosphere macerates (roots plus adhering soil) of rice (Oryza sativa cv. Huyêt Ròng) that was cultivated on a soil in the Bình Thanh region of Vietnam, which is $10 \mathrm{~km}$ northeast of Hô Chi Minh City (62). This soil is an acid sulfate soil (Thionic
Fluvisol according to the Food and Agriculture Organization classification) with a silty clay loam texture. $\mathrm{N}_{2}$-fixing bacteria were enumerated and isolated by using the procedure described previously by Omar et al. (42). Azospirillum lipoferum 4B (5) was used as a positive control for nitrogen fixation.

All strains were maintained on nutrient agar $(0.1 \%$ [wt/vol] beef extract, $0.2 \%$ [wt/vol] yeast extract, $0.5 \%$ [wt/vol] $\mathrm{NaCl}, 0.5 \%$ [wt/vol] peptone, $2.0 \%[\mathrm{wt} / \mathrm{vol}]$ agar; $\mathrm{pH}$ 7.4). The bacteriological purity of each strain was verified by plating and by microscopic examination of living and Gram-stained cells. When two or more colony types (colony types $\mathrm{t} 1, \mathrm{t} 2$, and $\mathrm{t} 3$ ) were found, they were compared by protein sodium dodecyl sulfate-polyacrylamide gel electrophoresis (47) in order to determine whether they were colony morphology variants. If two colony types produced almost identical protein patterns, only one type was included in this study. All strains were grown at $28^{\circ} \mathrm{C}$.

Biochemical tests. The following conventional tests were performed with the TVV strains: tests for pigment production on nutrient agar and King B medium (35), growth at temperatures between 20 and $41^{\circ} \mathrm{C}$ on nutrient agar, growth on PCAT medium (12), oxidase activity (49), and onion pathogenicity (26). The API 2ONE microtube system method (bioMérieux, La Balme-les-Grottes, France) was used as a standardized method to determine nitrate reduction, hydrolysis of gelatin and esculin, glucose fermentation, arginine dihydrolase activity, production of indole, $\beta$-galactosidase activity, and urease activity. Transmission electron microscopy studies were performed with strains TVV75 ${ }^{\mathrm{T}}$, TVV69, TVV70, and TVV71 to observe flagella by the methods described by Berge et al. (7).

Carbon substrate assimilation tests. API galleries (API 50CH, API 50AO, and API 50AA; bioMérieux) were used to determine the assimilation of 147 organic compounds as sole carbon sources. The experimental procedure which we used has been described previously (33). Nine strains were tested in duplicate on separate occasions to verify the reproducibility of the method.

Numerical analysis of phenotypic characteristics. The results of auxanographic tests were scored as described previously (33). A total of 147 characteristics were examined, and the results of tests for growth on inulin, L-sorbose, methyl-xyloside, esculin, isophthalate, gluconate, fumarate, L-malate, L-aspartate, and L-glutamate were not included in the numerical analysis because they were negative for all strains. The levels of interstrain similarity $(S)$ were calculated by using a similarity coefficient derived from the Canberra metric coefficient $\left(d_{\mathrm{CANB}}\right)(54)$ and the following equation: $S=100 \times\left(1-d_{\mathrm{CANB}}\right)$. A cluster analysis was performed by using the unweighted average pair group method (54), the Clustan version 2.1 program of Wishart (69), and the Siemens model 7570-C computer of the Akademisch Rekencentrum, Universiteit Gent, Ghent, Belgium.

Nitrogenase assay. The ability of each strain to fix $\mathrm{N}_{2}$ was estimated by using the acetylene reduction assay and an agar slant under an air atmosphere. Pure cultures of strains were streaked onto solid WAT4C medium slants (7) containing four carbon sources $(0.50 \%$ [wt/vol] starch, $0.50 \%$ [wt/vol] glucose, $0.50 \%$ [wt/vol] mannitol, $0.35 \%$ [wt/vol] malic acid). Uninoculated tubes were used as negative controls. The tubes were immediately closed with rubber stoppers (Suba-Seal), and acetylene $(1 \%, \mathrm{vol} / \mathrm{vol})$ was injected. Ethylene production was measured after 4 days of incubation at $28^{\circ} \mathrm{C}$ with a flame ionization gas chromatograph (Varian series 1400 aerograph).

Isolation of high-molecular-weight DNA. For DNA-rRNA hybridization experiments we used high-molecular-weight DNA prepared by the method of Marmur (39). Cells were grown for 2 to 3 days in Roux flasks on a solid medium as described above.

For DNA-DNA hybridization experiments high-molecular-weight DNA was extracted and purified by using the procedure of Brenner et al. (10).

DNA base composition. Mean $\mathrm{G}+\mathrm{C}$ contents were determined by using the thermal denaturation method (20) and were calculated by using the equation of Marmur and Doty (40), as modified by De Ley (17).

DNA-rRNA hybridization. DNA was purified by $\mathrm{CsCl}$ gradient centrifugation and was fixed on cellulose nitrate filters (type SM 11358; Sartorius, Göttingen, Germany) as described previously (19). The amount of filter-fixed DNA was determined chemically (66). ${ }^{3} \mathrm{H}$-labeled rRNAs from $B$. cepacia $\mathrm{LMG} 1222^{\mathrm{T}}$ and [A.] eutrophus LMG $1199^{\mathrm{T}}$ were prepared and purified by using the method described by De Ley and De Smedt (19). Cells were labeled in vivo by adding 0.8 $\mathrm{mCi}$ of $\left[2,8{ }^{-3} \mathrm{H}\right]$ adenine and $2.8 \mathrm{mCi}$ of $\left[5,6^{-3} \mathrm{H}\right]$ uracil (New England Nuclear Research Products, Boston, Mass.) in $150 \mathrm{ml}$ of medium containing $1 \%$ (wt/vol) glucose, $1 \%(\mathrm{wt} / \mathrm{vol})$ yeast extract, $0.1 \%\left(\mathrm{NH}_{4}\right)_{2} \mathrm{SO}_{4}$, and $0.025 \%(\mathrm{wt} / \mathrm{vol})$ $\mathrm{KH}_{2} \mathrm{PO}_{4}(\mathrm{pH} 7)$. The specific activities of the $23 \mathrm{~S}$ rRNA fractions were 7,000 cpm for B. cepacia LMG $1222^{\mathrm{T}}$ and $6,000 \mathrm{cpm}$ for [A.] eutrophus LMG $1199^{\mathrm{T}}$. Labeled rRNA from [B.] solanacearum NCPPB $325^{\mathrm{T}}$ was available from members of our research group (22). Each hybrid was characterized by its $T_{m(e)}$. $\Delta T_{m(e)}$ is the difference between $T_{m(e)}$ s of the homologous and the heterologous duplexes.

DNA-DNA hybridization and thermal stability of duplexes. Native DNA was labeled in vitro by nick translation with tritium-labeled nucleotides (Amersham International, Amersham, England). The procedure used for the hybridization experiments (S1 nuclease-trichloroacetic acid method) has been described previously (28). The reassociation temperature was $70^{\circ} \mathrm{C}$. DNA-DNA hybridization experiments were performed by using labeled DNAs from the following strains as probes: B. cepacia LMG $1222^{\mathrm{T}}$ and LMG 6964 and B. vietnamiensis LMG 6998 and TVV70. 
TABLE 1. Strains used

\begin{tabular}{|c|c|c|c|c|}
\hline Taxon & Strain $^{a}$ & Other designation $^{a}$ & Source of isolation & Phenon \\
\hline \multicolumn{5}{|l|}{ New isolates } \\
\hline Burkholderia vietnamiensis & TVV69 & LMG 10926 & Rice rhizosphere soil, Bình Thanh, Vietnam & II \\
\hline Burkholderia vietnamiensis & TVV70 & LMG 10927 & Rice rhizosphere soil, Bình Thanh, Vietnam & II \\
\hline Burkholderia vietnamiensis & TVV71 & LMG 10928 & Rice rhizosphere soil, Bình Thanh, Vietnam & II \\
\hline Burkholderia vietnamiensis & TVV75 ${ }^{\mathrm{T}}$ & LMG $10929^{\mathrm{T}}$ & Rice rhizosphere soil, Bình Thanh, Vietnam & II \\
\hline Burkholderia vietnamiensis & TVV116 & LMG 10930 & Rice rhizosphere soil, Bình Thanh, Vietnam & II \\
\hline Burkholderia vietnamiensis & TVV128 & LMG 10931 & Rice rhizosphere soil, Bình Thanh, Vietnam & II \\
\hline Burkholderia vietnamiensis & TVV135 & LMG 11346 & Rice rhizosphere soil, Bình Thanh, Vietnam & II \\
\hline Burkholderia vietnamiensis & TVV72 & LMG 11347 & Rice rhizosphere soil, Bình Thanh, Vietnam & II \\
\hline Burkholderia vietnamiensis & TVV115 & LMG 11348 & Rice rhizosphere soil, Bình Thanh, Vietnam & II \\
\hline Burkholderia vietnamiensis & TVV74 & LMG 11349 & Rice rhizosphere soil, Bình Thanh, Vietnam & II \\
\hline Burkholderia vietnamiensis & TVV127 & LMG 11350 & Rice rhizosphere soil, Bình Thanh, Vietnam & II \\
\hline Burkholderia vietnamiensis & TVV131 & LMG 10932 & Rice rhizosphere soil, Bình Thanh, Vietnam & \\
\hline \multicolumn{5}{|l|}{ Collection strains } \\
\hline Burkholderia vietnamiensis & LMG 6998 & CCUG 7246 & Blood & II \\
\hline Burkholderia vietnamiensis & LMG 6999 & CCUG 9631 & Neck abscess, child & II \\
\hline Burkholderia cepacia & LMG 11351 & PHP7 & Maize rhizosphere & I \\
\hline Burkholderia cepacia & LMG $1222^{\mathrm{T}}$ & ATCC $25416^{\mathrm{T}}$ & Allium cepa $\mathrm{L}$. & I \\
\hline Burkholderia cepacia & LMG 1275 & Stanier 382 & Forest soil & I \\
\hline Burkholderia cepacia & LMG 6032 & CCUG 787 & & I \\
\hline Burkholderia cepacia & LMG 6859 & NCIB 9087 & Rotting tree trunk & I \\
\hline Burkholderia cepacia & LMG $6860 \mathrm{t} 1^{b}$ & NCIB 9091t1 & Soil & I \\
\hline Burkholderia cepacia & LMG $6861 \mathrm{t} 1$ & NCIB $9092 \mathrm{t} 1$ & Soil & I \\
\hline Burkholderia cepacia & LMG 6862t1 & NCIB 9093t1 & Forest soil & I \\
\hline Burkholderia cepacia & LMG 6863t1 & NCIB 9690t1 & River Maraval & I \\
\hline Burkholderia cepacia & LMG 6864 & NCIB 9691 & Soil enriched with D-mandelate & I \\
\hline Burkholderia cepacia & LMG 6865 & NCIB 10649 & & I \\
\hline Burkholderia cepacia & LMG 6887 & NCPPB 945 & & I \\
\hline Burkholderia cepacia & LMG 6888 & NCPPB 946 & & I \\
\hline Burkholderia cepacia & LMG 6889t1 & NCPPB 1962t1 & & I \\
\hline Burkholderia cepacia & LMG 6959 & PDDCC 3177 & Allium cepa $\mathrm{L}$. & I \\
\hline Burkholderia cepacia & LMG 6960t1 & PDDCC 5837t1 & & I \\
\hline Burkholderia cepacia & LMG 6961t1 & PDDCC 5841t1 & & I \\
\hline Burkholderia cepacia & LMG 6962 & PDDCC 5952 & Allium cepa $\mathrm{L}$. & I \\
\hline Burkholderia cepacia & LMG 6963t1 & ATCC $17460 t 1$ & Soil & I \\
\hline Burkholderia cepacia & LMG 6964 & PDDCC 5982 & Lycopersicon lycopersicum (L.) Karst. ex Farw. & I \\
\hline Burkholderia cepacia & LMG 6980 & CNBP 1434 & Allium cepa $\mathrm{L}$. & I \\
\hline Burkholderia cepacia & LMG 6981t1 & ATCC $25609 t 1$ & Bronchial washings & I \\
\hline Burkholderia cepacia & LMG 6981t2 & ATCC $25609 \mathrm{t} 2$ & Bronchial washings & I \\
\hline Burkholderia cepacia & LMG 6981t3 & ATCC $25609 \mathrm{t} 3$ & Bronchial washings & I \\
\hline Burkholderia cepacia & LMG 6986 & CCUG 788 & Urine & I \\
\hline Burkholderia cepacia & LMG 6987t1 & CCUG $853 \mathrm{t} 1$ & & I \\
\hline Burkholderia cepacia & LMG 6988 & CCUG 1603 & Leg wound & I \\
\hline Burkholderia cepacia & LMG 6989t1 & CCUG 2393t1 & Blood & I \\
\hline Burkholderia cepacia & LMG 6990t1 & CCUG 2851t1 & & I \\
\hline Burkholderia cepacia & LMG $6991 \mathrm{t} 1$ & CCUG $2856 \mathrm{t} 1$ & Forest soil & I \\
\hline Burkholderia cepacia & LMG 6992 & CCUG 2857 & Soil & I \\
\hline Burkholderia cepacia & LMG 6993 & DSM 50180 & Soil & I \\
\hline Burkholderia cepacia & LMG 6994 & CCUG 2859 & Forest soil & I \\
\hline Burkholderia cepacia & LMG 6996 & CCUG 3000 & Urine & I \\
\hline Burkholderia cepacia & LMG 6997 & CCUG 3461B & Ear & I \\
\hline Burkholderia cepacia & LMG 7000 & CCUG 13348 & Blood & I \\
\hline Burkholderia cepacia & LMG 6995 & CCUG 1440 & Urine & $\operatorname{sep}^{c}$ \\
\hline Burkholderia gladioli pv. alliicola & LMG $2121^{d}$ & NCPPB $947^{d}$ & & III \\
\hline Burkholderia gladioli pv. alliicola & LMG 6877 & NCPPB 948 & & III \\
\hline Burkholderia gladioli pv. alliicola & LMG 6878 & NCPPB 2478 & Allium cepa $\mathbf{L}$. & III \\
\hline Burkholderia gladioli pv. alliicola & LMG 6915 & NRRL B-828 & & III \\
\hline Burkholderia gladioli pv. alliicola & LMG 6954 & PDDCC 3410 & Allium cepa $\mathrm{L}$. & III \\
\hline Burkholderia gladioli pv. alliicola & LMG 6955 & PDDCC 3411 & Allium cepa $\mathrm{L}$. & III \\
\hline Burkholderia gladioli pv. alliicola & LMG 6956 & PDDCC 3412 & Allium cepa $\mathrm{L}$. & III \\
\hline Burkholderia gladioli pv. alliicola & LMG 6957 & PDDCC 5838 & & III \\
\hline Burkholderia gladioli pv. alliicola & LMG 6958 & PDDCC 5840 & & III \\
\hline Burkholderia gladioli pv. alliicola & LMG 6979 & CNBP 1435 & Allium cepa $\mathrm{L}$. & III \\
\hline Burkholderia gladioli pv. gladioli & LMG $2216^{\mathrm{T}}$ & ATCC $10248^{\mathrm{T}}$ & Gladiolus sp. & III \\
\hline Burkholderia gladioli pv. gladioli & LMG 6880 & NCPPB 644 & Gladiolus sp. & III \\
\hline Burkholderia gladioli pv. gladioli & LMG 6881 & NCPPB 1051 & Gladiolus sp. & III \\
\hline Burkholderia gladioli pv. gladioli & LMG 6882 & NCPPB 1887 & Gladiolus sp. & III \\
\hline Burkholderia gladioli pv. gladioli & LMG 6884 & NCPPB 1890 & Gladiolus sp. & III \\
\hline
\end{tabular}


TABLE 1-Continued

\begin{tabular}{|c|c|c|c|c|}
\hline Taxon & Strain $^{a}$ & Other designation $^{a}$ & Source of isolation & Phenon \\
\hline Burkholderia caryophylli & LMG $2155^{\mathrm{T}}$ & ATCC $25418^{\mathrm{T}}$ & Dianthus caryophyllus L. & IV \\
\hline Burkholderia caryophylli & LMG 2156 & NCPPB 609 & Dianthus caryophyllus L. & IV \\
\hline Burkholderia caryophylli & LMG 2157 & NCPPB 349 & Dianthus caryophyllus L. & IV \\
\hline Burkholderia caryophylli & LMG 6876 & NCPPB 353 & Dianthus sp. & IV \\
\hline Burkholderia caryophylli & LMG 6978 & CNBP 1370 & Dianthus caryophyllus L. & IV \\
\hline Burkholderia glumae & LMG $2196^{\mathrm{T}}$ & NCPPB $2981^{\mathrm{T}}$ & Oryza sativa $\mathrm{L}$. & $\mathrm{V}$ \\
\hline Burkholderia glumae & LMG 1277 & NCPPB 2391 & Oryza sativa $\mathrm{L}$. & $\mathrm{V}$ \\
\hline Burkholderia glumae & LMG 6952 & PDDCC 3728 & Oryza sativa $\mathrm{L}$. & $\mathrm{V}$ \\
\hline Burkholderia plantarii & LMG $9035^{\mathrm{T}}$ & ICMP $9424^{\mathrm{T}}$ & Oryza sativa $\mathrm{L}$. & VI \\
\hline Burkholderia plantarii & LMG 10908 & ICMP 9426 & Oryza sativa $\mathrm{L}$. & VI \\
\hline [Pseudomonas] phenazinium & LMG $2247^{\mathrm{T}}$ & NCIB $11027^{\mathrm{T}}$ & Soil enriched with threonine & VII \\
\hline [Pseudomonas $]$ phenazinium & LMG 6868t1 & NCIB 11431t1 & & VII \\
\hline [Pseudomonas] phenazinium & LMG $6868 \mathrm{t} 2$ & NCIB 11431t2 & & VII \\
\hline Burkholderia andropogonis & LMG 2126 & NCPPB 450 & Trifolium repens & VIII \\
\hline Burkholderia andropogonis & LMG 2127 & NCPPB 451 & Trifolium pratense $\mathrm{L}$. & VIII \\
\hline Burkholderia andropogonis & LMG 2128 & NCPPB 933 & Zea mays $\mathrm{L}$ & VIII \\
\hline Burkholderia andropogonis & LMG $2129^{\mathrm{T}}$ & NCPPB $934^{\mathrm{T}}$ & Sorghum bicolor (L.) Moench & VIII \\
\hline Burkholderia andropogonis & LMG 2327 & Hayward 0364A & Trifolium repens $\mathbf{L}$. & VIII \\
\hline Burkholderia andropogonis & LMG 2329 & NCPPB 1024 & Mucuna deeringianum (Bort) Merrill & VIII \\
\hline Burkholderia andropogonis & LMG 6872 & NCPPB 1127 & Bougainvillea sp. & VIII \\
\hline Burkholderia andropogonis & LMG 6873 & NCPPB 1693 & Rheum rhaponticum $\mathrm{L}$. & VIII \\
\hline Burkholderia andropogonis & LMG 6874 & NCPPB 2179 & Sorghum bicolor (L.) Moench & VIII \\
\hline Burkholderia andropogonis & LMG 6875 & NCPPB 2868 & Trifolium repens $\mathrm{L}$. & VIII \\
\hline Burkholderia andropogonis & LMG 6942 & PDDCC 3994 & Mucuna deeringianum (Bort) Merrill & VIII \\
\hline Burkholderia andropogonis & LMG 6943 & PDDCC 6779 & Coffea arabica $\mathrm{L}$. & VIII \\
\hline Burkholderia andropogonis & LMG 6944 & PDDCC 6780 & Coffea arabica $\mathrm{L}$. & VIII \\
\hline Burkholderia andropogonis & LMG 6945 & PDDCC 5980 & Sorghum bicolor (L.) Moench & VIII \\
\hline Burkholderia andropogonis & LMG 6946 & PDDCC 7076 & Cicer arietinum $\mathrm{L}$. & VIII \\
\hline Burkholderia andropogonis ${ }^{e}$ & LMG 1279 & NCPPB 2441 & Setcreasea pallida Rose & VIII \\
\hline Burkholderia andropogonis $s^{e}$ & LMG $2362^{f}$ & NCPPB 968 & Dianthus caryophyllus L. & VIII \\
\hline Burkholderia andropogonis ${ }^{e}$ & LMG 2363 & NCPPB 2157 & Dianthus barbatus L. & VIII \\
\hline Burkholderia andropogonis ${ }^{e}$ & LMG 6947 & PDDCC 4001 & Dianthus sp. & VIII \\
\hline Burkholderia andropogonis ${ }^{e}$ & LMG 6948 & PDDCC 4003 & Dianthus sp. & VIII \\
\hline Burkholderia andropogonise & LMG 6949 & PDDCC 6647 & Dianthus caryophyllus L. & VIII \\
\hline Burkholderia andropogonis ${ }^{e}$ & LMG 6950 & PDDCC 6649 & Dianthus caryophyllus L. & VIII \\
\hline [Burkholderia] solanacearum & LMG 2295 & NCPPB 173 & Solanum tuberosum $\mathbf{L}$. & sep \\
\hline [Burkholderia] solanacearum & LMG 2296 & NCPPB 215 & Lycopersicon lycopersicum (L.) Karst. ex Farw. & IX \\
\hline [Burkholderia] solanacearum & LMG 2297 & NCPPB 253 & Casuarina equisetifolia J. R. Forst. and G. Forst & IX \\
\hline [Burkholderia] solanacearum & LMG 2298 & NCPPB 282 & Solanum tuberosum L. & IX \\
\hline [Burkholderia] solanacearum & LMG $2299^{\mathrm{T}}$ & NCPPB $325^{\mathrm{T}}$ & Lycopersicon lycopersicum (L.) Karst. ex. Farw. & IX \\
\hline [Burkholderia] solanacearum & LMG 2300 & NCPPB 339 & Solanum tuberosum $\mathrm{L}$. & IX \\
\hline [Burkholderia] solanacearum & LMG 2301 & NCPPB 446 & Musa sp. & IX \\
\hline [Burkholderia] solanacearum & LMG 2303 & NCPPB 789 & Musa sp. & IX \\
\hline [Burkholderia] solanacearum & LMG 2304 & NCPPB 792 & Tectona grandis & IX \\
\hline [Burkholderia] solanacearum & LMG 2305 & NCPPB 909 & Solanum tuberosum $\mathrm{L}$. & IX \\
\hline [Burkholderia] solanacearum & LMG 2306 & NCPPB 1019 & Lycopersicon lycopersicum (L.) Karst. ex Farw. & IX \\
\hline [Burkholderia] solanacearum & LMG 5839 & NCPPB 1579 & Zingiber officinale & IX \\
\hline [Alcaligenes] eutrophus & LMG $1199^{\mathrm{T}}$ & ATCC $17697^{\mathrm{T}}$ & & $\mathrm{X}$ \\
\hline [Alcaligenes] eutrophus & LMG 1200 & ATCC 17698 & & $\mathrm{X}$ \\
\hline [Alcaligenes] eutrophus & LMG 1190 & ATCC 33178 & & $\mathrm{X}$ \\
\hline [Alcaligenes] eutrophus & LMG 1210 & ATCC 17710 & & $\mathrm{X}$ \\
\hline [Alcaligenes] eutrophus & LMG 1211 & ATCC 25207 & & $\mathrm{X}$ \\
\hline [Alcaligenes] eutrophus & LMG 1206 & ATCC 17706 & & $\mathrm{X}$ \\
\hline [Alcaligenes] eutrophus & LMG 1207 & ATCC 17707 & & $\mathrm{X}$ \\
\hline [Alcaligenes] eutrophus & LMG 1201 & ATCC 17699 & & \\
\hline [Alcaligenes] eutrophus & LMG 1202 & ATCC 17700 & & \\
\hline [Alcaligenes] eutrophus & LMG 1203 & ATCC 17702 & & \\
\hline [Alcaligenes] eutrophus & LMG 1204 & ATCC 17704 & & \\
\hline [Alcaligenes] eutrophus & LMG 1205 & ATCC 17705 & & \\
\hline [Alcaligenes] eutrophus & LMG 1209 & ATCC 17709 & & \\
\hline "Pseudomonas oxalaticus" & LMG $2235^{g}$ & NCIB $8642^{g}$ & Indian earthworm & $\mathrm{X}$ \\
\hline "Pseudomonas oxalaticus" & LMG 2233 & NCIB 8543 & Indian earthworm & $\mathrm{X}$ \\
\hline "Pseudomonas oxalaticus" & LMG 2234t1 & NCIB $8544 t 1$ & Indian earthworm & $X$ \\
\hline "Pseudomonas oxalaticus" & LMG 2236 & NCIB 8643 & Indian earthworm & $\mathrm{X}$ \\
\hline [Burkholderia] pickettii & LMG $5942^{\mathrm{T}}$ & ATCC $27511^{\mathrm{T}}$ & Tracheotomy patient & XI \\
\hline [Burkholderia] pickettii & LMG 6866 & NCIB 10805 & & XI \\
\hline [Burkholderia] pickettii & LMG 6871 & CCM 2846 & Soil of a rice field & XI \\
\hline [Burkholderia] pickettii & LMG 7001 & CCUG 3314 & & XI \\
\hline
\end{tabular}


TABLE 1-Continued

\begin{tabular}{|c|c|c|c|c|c|}
\hline & Taxon & Strain $^{a}$ & Other designation ${ }^{a}$ & Source of isolation & Phenon \\
\hline [Burkholderia] & pickettii & LMG 7002 & CCUG 3316 & & XI \\
\hline [Burkholderia] & pickettii & LMG 7003 & CCUG 3317 & & XI \\
\hline [Burkholderia] & pickettii & LMG 7004 & CCUG 3319 & & XI \\
\hline [Burkholderia] & pickettii & LMG 7005 & CCUG 1467 & & XI \\
\hline [Burkholderia] & pickettii & LMG 7006 & CCUG 1742 & Blood & XI \\
\hline [Burkholderia] & pickettii & LMG 7007 & CCUG 3324 & & XI \\
\hline [Burkholderia] & pickettii & LMG 7008 & CCUG 2165 & Urine & XI \\
\hline [Burkholderia] & pickettii & LMG 7009 & CCUG 8527 & Trachea & XI \\
\hline [Burkholderia] & pickettii & LMG 7010 & CCUG 9984 & Urethra & XI \\
\hline [Burkholderia] & pickettii & LMG 7011 & CCUG 11629 & Trachea & XI \\
\hline Burkholderia] & pickettii & LMG 7012 & CCUG 12413 & & XI \\
\hline [Burkholderia] & pickettii & LMG 7013 & CCUG 12490 & Blood & XI \\
\hline [Burkholderia] & pickettii & LMG 7014 & CCUG 12491 & Blood & XI \\
\hline [Burkholderia] & pickettii & LMG 7015 & CCUG 12492 & Blood & XI \\
\hline [Burkholderia] & pickettii & LMG 7016 & CCUG 12493 & Blood & XI \\
\hline [Burkholderia] & pickettii & LMG 7017 & CCUG 12494 & Blood & XI \\
\hline [Burkholderia] & pickettii & LMG 7018 & CCUG 3323 & & XI \\
\hline [Burkholderia] & pickettii & LMG 7056 & CCUG 3321 & & XI \\
\hline [Burkholderia] & pickettii & LMG 7057 & CCUG 691 & Drain fluid & XI \\
\hline [Burkholderia] & pickettii & LMG 7058 & CCUG 3325 & & XI \\
\hline [Burkholderia] & pickettii & LMG 7140 & Pickett K-638 & & XI \\
\hline [Burkholderia] & pickettii & LMG 7141 & Pickett K-1303 & & XI \\
\hline [Burkholderia] & pickettii & LMG 7142 & Pickett K-1466 & & XI \\
\hline [Burkholderia] & pickettii & LMG 7143 & Pickett K-1503 & & XI \\
\hline [Burkholderia] & pickettii & LMG 7144 & Pickett K-1510 & & XI \\
\hline [Burkholderia] & pickettii & LMG 7145 & Pickett K-232 & & $\mathrm{XI}$ \\
\hline [Burkholderia] & pickettii & LMG 7147t1 & Pickett K-1193t1 & & XI \\
\hline [Burkholderia] & pickettii & LMG 7159 & CIP 73.24 & & XI \\
\hline [Burkholderia] & pickettii & LMG 7160 & CIP 74.22 & Blood & $\mathrm{XI}$ \\
\hline Burkholderia $c$ & cocovenenans & LMG $11626^{\mathrm{T}}$ & ATCC $33664^{\mathrm{T}}$ & & \\
\hline Brevundimona & as diminuta & LMG $2089^{\mathrm{T}}$ & ATCC $11568^{\mathrm{T}}$ & & \\
\hline Pseudomonas & aeruginosa & LMG 6395 & DSM 1117 & & \\
\hline Pseudomonas & stutzeri & LMG $2333^{\mathrm{T}}$ & ATCC $17588^{\mathrm{T}}$ & & \\
\hline Pseudomonas & putida & LMG $2257^{\mathrm{T}}$ & ATCC $12633^{\mathrm{T}}$ & & \\
\hline Sphingomonas & s paucimobilis & LMG $1227^{\mathrm{T}}$ & ATCC $29837^{\mathrm{T}}$ & & \\
\hline Telluria mixta & & LMG $11547^{\mathrm{T}}$ & UQM $1762^{\mathrm{T}}$ & Black earth soil & \\
\hline Azospirillum $l_{i}$ & ipoferum & LMG 4348 & $4 \mathrm{~B}$ & Rice rhizosphere (Camargue, France) & \\
\hline
\end{tabular}

${ }^{a}$ ATCC, American Type Culture Collection, Rockville, Md.; CCUG, Culture Collection of the University of Göteborg, Department of Clinical Bacteriology, University of Göteborg, Göteborg, Sweden; CIP, Collection de I'Institut Pasteur, Paris, France; CNBP, Collection Nationale de Bactéries Phytopathogènes, Institut National de la Recherche Agronomique, Station de Pathologie Végétale et Phytobactériologie, Angers, France; DSM, Deutsche Sammlung von Microorganismen, Braunschweig, Germany; LMG, Culture Collection, Laboratorium voor Microbiologie, State University of Ghent, Ghent, Belgium; NCIB, National Collection of Industrial Bacteria, Aberdeen, Scotland; NCPPB, National Collection of Plant Pathogenic Bacteria, Hatching Green, England; NRRL, Agricultural Research Service Culture Collection, Peoria, Ill.; PDDCC, Culture Collection of the Plant Diseases Division, New Zealand Department of Scientific and Industrial Research, Auckland, New Zealand; TVV, Tran Van Van; UQM, Department of Microbiology, University of Queensland, St. Lucia, Queensland, Australia.

${ }^{b}$ The suffixes $\mathrm{t} 1, \mathrm{t} 2$, and $\mathrm{t} 3$ indicate colony morphology variants.

${ }^{c}$ sep, separate position.

a Pathovar reference strain.

e Originally named [Pseudomonas] woodsii.

${ }^{f}$ Type strain of the former species [Pseudomonas] woodsii

g Co-type strain.

The thermal stability of reassociated DNAs was estimated by determining the denaturation temperature $\left(T_{m}\right)$ (the temperature at which $50 \%$ of the doublestranded DNA was denatured and lysed by $\mathrm{S} 1$ nuclease). The $T_{m}$ was determined by using the method of Crosa et al. (16). The divergence between DNAs was estimated by determining the $\Delta T_{m}$ value (the difference between the $T_{m}$ of the heteroduplex [in the heterologous reaction] and the $T_{m}$ of the homoduplex [in the homologous reaction]).

\section{RESULTS}

Phenotypic characteristics of the TVV strains. The number of natural nitrogen-fixing bacteria associated with the rhizosphere of rice plants grown in an acid sulfate soil in Bình Thanh, Vietnam, was $2.8 \times 10^{8}$ cells per $\mathrm{g}$ (dry weight) of rhizosphere soil. Among these nitrogen-fixing bacteria, the 12 TVV strains were dominant. These strains were motile at $28^{\circ} \mathrm{C}$, and polar flagella were observed when transmission electron microscopy was used. They grew well at $28^{\circ} \mathrm{C}$, and maximum growth was observed at $41^{\circ} \mathrm{C}$. Colonies on nutrient agar were smooth, translucent, and convex with entire margins, and colonies were not pigmented either on nutrient agar or on King B medium. Colonies on PCAT medium were white and smaller than $B$. cepacia LMG $1222^{\mathrm{T}}$ colonies. Nitrate was reduced to nitrite, $\beta$-galactosidase and oxidase were produced, and gelatin was hydrolyzed, but esculin was not hydrolyzed. Glucose fermentation, production of indole, arginine dihydrolase activity, and urease activity were negative. The TVV strains were not pathogenic for onions.

The TVV strains produced three types of ornibactins (41), which are new types of siderophores (60), but did not produce pyochelin or cepabactin, two compounds which are typically produced by $B$. cepacia strains. 
TABLE 2. Results of auxanographic studies

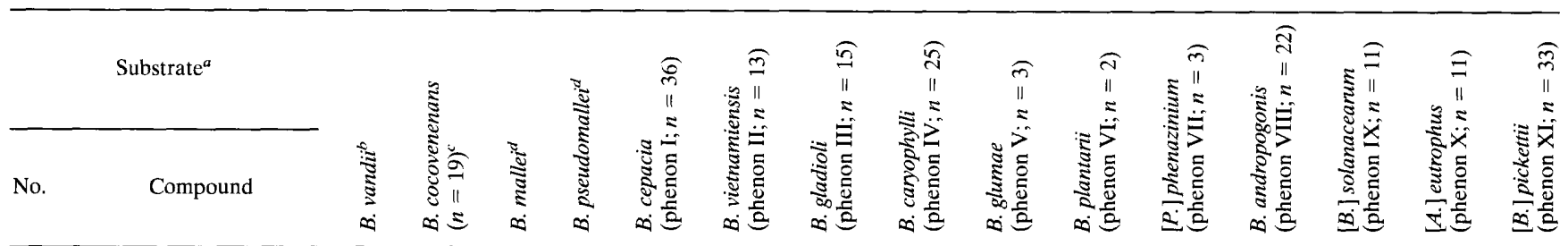

\begin{tabular}{|c|c|c|c|c|c|c|c|c|c|c|c|c|c|c|c|c|}
\hline 1 & Pyruvate & & $+^{e}$ & + & + & + & + & + & + & + & + & + & + & + & $\mathrm{d}^{-}$ & + \\
\hline 2 & D-Glucose & + & + & + & + & + & + & + & + & + & + & + & + & + & - & + \\
\hline 3 & Succinate & & + & + & + & + & + & + & + & + & + & + & + & + & + & + \\
\hline 4 & Glycerol & + & + & + & + & + & + & + & + & + & + & + & + & + & + & + \\
\hline 5 & $L-\alpha$-Alanine & & + & + & + & + & + & + & + & + & + & + & + & + & + & + \\
\hline 6 & Inositol & + & + & + & + & + & + & + & + & + & + & + & + & $\mathrm{d}^{+}$ & - & - \\
\hline 7 & Acetate & & + & + & + & + & + & + & + & + & + & + & + & $\mathrm{d}^{+}$ & + & + \\
\hline 8 & D- $\alpha$-Alanine & & + & + & + & + & + & + & + & + & + & + & + & $\mathrm{d}^{+}$ & + & + \\
\hline 9 & D-Galactose & + & + & + & + & + & + & + & + & + & + & + & + & $\mathrm{d}^{-}$ & - & + \\
\hline 10 & Sorbitol & + & + & + & + & + & + & + & + & + & + & + & + & $\mathrm{d}^{-}$ & - & - \\
\hline 11 & Mannitol & + & + & + & + & + & + & + & + & + & + & + & + & $d^{-}$ & - & $d^{-}$ \\
\hline 12 & DL-Lactate & & + & + & + & + & + & + & + & + & + & + & + & $\mathrm{d}^{+}$ & + & + \\
\hline 13 & L-Threonine $f$ & + & + & + & + & + & + & + & + & + & + & + & - & + & + & + \\
\hline 14 & L-Histidine ${ }^{f}$ & & + & + & + & + & + & + & + & + & + & + & - & + & + & + \\
\hline 15 & L-Tyrosine $\mathrm{e}^{f}$ & & + & + & + & + & + & + & + & + & + & + & - & + & + & + \\
\hline 16 & Trehalose & + & + & + & + & + & + & + & + & + & + & - & $d^{-}$ & $d^{-}$ & - & $d^{-}$ \\
\hline 17 & D-Mannose & + & & + & + & + & + & + & + & + & + & + & + & - & - & - \\
\hline 18 & DL-Glycerate & & & + & + & + & + & + & + & + & + & + & + & + & + & + \\
\hline 19 & $p$-Hydroxybenzoate & & & + & + & + & + & + & + & + & + & + & + & $d^{-}$ & $\mathrm{d}^{-}$ & + \\
\hline 20 & DL-4-Aminobutyrate & & & + & + & + & + & + & + & + & + & + & $\mathrm{d}^{+}$ & + & $\mathrm{d}^{+}$ & + \\
\hline 21 & Betaine & & & + & + & + & + & + & + & + & + & + & - & - & - & - \\
\hline 22 & Propionate $f$ & & & + & + & + & + & + & + & + & + & $\mathrm{d}^{+}$ & - & $d^{+}$ & + & + \\
\hline 23 & L-Valine $\mathrm{f}^{f}$ & + & - & $\mathrm{d}$ & + & + & + & + & + & + & + & + & - & + & $\mathrm{d}^{+}$ & + \\
\hline 24 & L-Proline & & + & $\mathrm{d}$ & + & + & + & + & + & + & + & + & + & + & + & + \\
\hline 25 & Citrate $^{f}$ & - & + & $\mathrm{d}$ & + & + & + & + & + & + & + & + & + & + & + & + \\
\hline 26 & L-Serine & & + & $\mathrm{d}$ & + & + & + & + & + & + & + & + & + & + & + & + \\
\hline 27 & 2-Ketogluconate & & & $\mathrm{d}$ & + & + & + & + & + & + & + & + & $\mathrm{d}^{-}$ & - & $\mathrm{d}^{+}$ & + \\
\hline 28 & L-Phenylalanine ${ }^{f}$ & & $d^{-}$ & d & + & + & + & + & + & + & + & + & - & $d^{-}$ & + & + \\
\hline 29 & D-Fructose & & + & $\mathrm{d}$ & + & + & + & + & + & + & + & + & + & + & $d^{+}$ & + \\
\hline 30 & D-Arabitol & & & & & + & + & + & + & + & + & + & + & $\mathrm{d}^{-}$ & - & $d^{--}$ \\
\hline 31 & DL-3-Hydroxybutyrate & & + & & & + & + & + & + & + & + & + & $d^{+}$ & + & + & + \\
\hline 32 & L-Arabinose & & + & $\mathrm{d}$ & - & + & + & + & + & + & + & + & + & - & - & + \\
\hline 33 & D-Malate & & & $\mathrm{d}$ & d & + & + & + & + & + & + & - & + & - & + & + \\
\hline 34 & Isobutyrate ${ }^{f}$ & & - & - & + & + & + & + & + & + & + & - & - & $\mathrm{d}^{+}$ & $\mathrm{d}^{+}$ & + \\
\hline 35 & D-Lyxose ${ }^{f}$ & & - & & & + & + & + & + & + & + & $\mathrm{d}^{+}$ & + & - & - & - \\
\hline 36 & Butyrate ${ }^{f}$ & & + & $\mathrm{d}$ & + & + & + & + & + & + & + & $\mathrm{d}^{+}$ & - & $\mathrm{d}^{+}$ & + & + \\
\hline 37 & Glucosamine ${ }^{f}$ & & & & & + & + & + & + & + & + & $\mathrm{d}^{+}$ & - & - & - & + \\
\hline 38 & Aconitate $^{f}$ & & + & - & + & + & + & + & + & + & $d^{+}$ & + & - & - & $\mathrm{d}^{-}$ & + \\
\hline 39 & L-Isoleucine $f^{f}$ & & - & - & + & + & + & + & + & + & $\mathrm{d}^{-}$ & + & - & + & + & + \\
\hline 40 & L-Arginine ${ }^{f}$ & & + & + & + & + & + & + & + & + & $\mathrm{d}^{-}$ & + & - & - & - & - \\
\hline 41 & $N$-Acetylglucosamine ${ }^{f}$ & & & + & & + & + & + & + & + & + & + & - & - & $\mathrm{d}^{+}$ & $\mathrm{d}^{+}$ \\
\hline 42 & L-Leucine ${ }^{f}$ & & - & - & - & + & + & + & + & $\mathrm{d}^{+}$ & + & $\mathrm{d}^{+}$ & - & $\mathrm{d}^{+}$ & + & + \\
\hline 43 & L-Tryptophanf & & - & + & + & + & + & + & + & + & - & + & - & $\mathrm{d}^{+}$ & + & + \\
\hline 44 & Sarcosine $\mathrm{e}^{f}$ & & + & $\mathrm{d}$ & d & + & + & + & + & $d^{+}$ & - & $d^{+}$ & - & $d^{-}$ & - & - \\
\hline 45 & $\beta$-Alanine $f^{f}$ & & + & + & + & + & + & + & + & $\mathrm{d}^{+}$ & - & + & - & $\mathrm{d}^{+}$ & + & + \\
\hline 46 & $n$-Valerate ${ }^{f}$ & & & - & + & + & + & + & $d^{-}$ & - & - & - & - & $\mathrm{d}^{+}$ & + & + \\
\hline 47 & L-Ornithine $f$ & + & - & - & + & + & + & + & + & - & - & - & - & $\mathrm{d}^{-}$ & $\mathrm{d}^{-}$ & $d^{+}$ \\
\hline 48 & L-Norleucine ${ }^{f}$ & & - & - & - & + & + & + & $d^{-}$ & - & - & $\mathrm{d}^{+}$ & - & - & + & $\mathrm{d}^{+}$ \\
\hline 49 & L-Lysine ${ }^{f}$ & & + & - & + & + & + & + & $\mathrm{d}^{-}$ & - & - & $\mathrm{d}^{+}$ & - & - & - & - \\
\hline 50 & DL-Kynurenine ${ }^{f}$ & & & - & + & + & + & + & $\mathrm{d}^{-}$ & - & - & $\mathrm{d}^{+}$ & $\mathrm{d}^{-}$ & $\mathrm{d}^{+}$ & $\mathrm{d}$ & $d^{+}$ \\
\hline 51 & $n$-Caproate $f$ & - & & - & + & + & + & + & - & - & $\mathrm{d}^{+}$ & - & - & - & + & + \\
\hline 52 & Heptanoate $f^{f}$ & + & & - & + & + & + & + & - & + & + & + & - & - & + & + \\
\hline 53 & Caprylate $^{f}$ & + & + & - & & + & + & + & - & $d^{-}$ & + & + & - & - & + & $d^{+}$ \\
\hline 54 & Levulinate $f$ & - & - & - & + & + & + & + & - & - & - & + & - & $\mathrm{d}^{+}$ & + & + \\
\hline 55 & Pelargonate $f^{f}$ & & + & - & + & + & + & + & - & $\mathrm{d}^{+}$ & + & + & - & - & + & $d^{-}$ \\
\hline 56 & Caprate & + & & - & + & + & + & + & - & + & + & $d^{+}$ & - & - & $d^{-}$ & $d^{-}$ \\
\hline 57 & DL-5-Aminovalerate $f$ & & & & & + & + & + & - & - & - & - & - & - & $\mathrm{d}^{-}$ & - \\
\hline 58 & Ethanolamine ${ }^{f}$ & - & + & - & + & + & + & + & - & + & + & + & $\mathrm{d}^{+}$ & - & - & - \\
\hline 59 & 2-Aminobenzoate ${ }^{f}$ & & & - & - & + & + & + & - & + & - & + & $\mathrm{d}^{-}$ & - & $\mathrm{d}^{-}$ & $\mathrm{d}^{-}$ \\
\hline 60 & Adipate $f^{f}$ & & + & + & + & + & + & + & - & - & - & $\mathrm{d}^{+}$ & - & - & $\mathrm{d}^{+}$ & $\mathrm{d}^{+}$ \\
\hline 61 & Azelate & - & + & $\mathrm{d}$ & $\mathrm{d}$ & + & + & + & + & + & + & + & - & + & + & + \\
\hline
\end{tabular}


TABLE 2-Continued

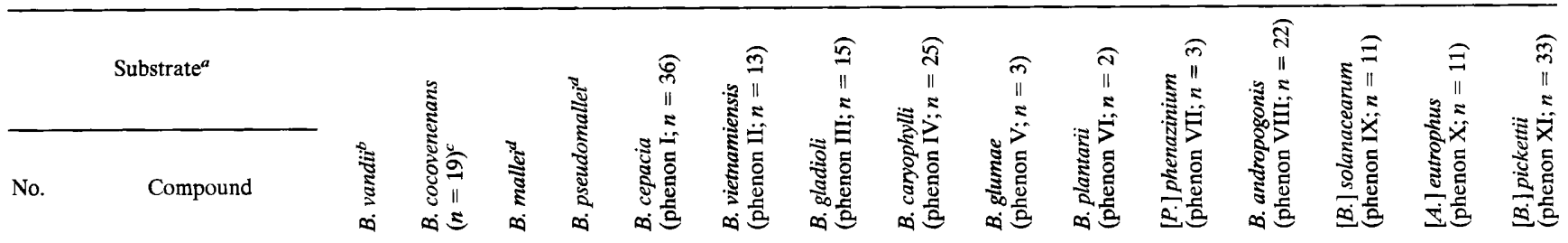

\begin{tabular}{|c|c|c|c|c|c|c|c|c|c|c|c|c|c|c|c|c|}
\hline 62 & Sebacate $^{f}$ & & + & - & + & + & + & + & + & + & + & + & - & $d^{+}$ & + & + \\
\hline 63 & L-Cysteine $f$ & & & & & + & + & + & + & + & + & + & + & $d^{+}$ & $\mathrm{d}^{-}$ & + \\
\hline 64 & Phenylacetate $^{f}$ & & & + & + & + & + & + & - & - & - & + & - & - & + & $d^{-}$ \\
\hline 65 & Dulcitof & & & & & + & + & + & - & + & + & - & - & $\mathrm{d}^{-}$ & - & - \\
\hline 66 & D-Arabinose $\mathrm{e}^{f}$ & & & + & + & + & + & + & + & + & + & + & + & - & - & - \\
\hline 67 & D-Tagatose ${ }^{f}$ & & & & & + & + & + & - & $\mathrm{d}^{+}$ & - & - & - & - & - & - \\
\hline 68 & D-Fucose ${ }^{f}$ & & - & + & + & + & + & + & + & $d^{+}$ & + & $d^{+}$ & $d^{-}$ & - & - & + \\
\hline 69 & L-Fucose $\mathrm{f}^{f}$ & & & & & + & + & + & + & + & + & + & - & - & - & - \\
\hline 70 & Amygdalin $f$ & & & & & + & $d^{+}$ & - & - & $d^{-}$ & + & - & - & - & - & - \\
\hline 71 & Glutarate $^{f}$ & - & - & d & $\mathrm{d}$ & + & + & $\mathrm{d}^{+}$ & - & - & - & - & - & - & + & $d^{+}$ \\
\hline 72 & Ribose $^{f}$ & & + & - & + & + & $\mathrm{d}^{-}$ & + & + & + & + & + & + & - & - & $d^{-}$ \\
\hline 73 & Malonate $f$ & & + & d & - & + & $\mathrm{d}^{-}$ & + & - & $d^{-}$ & $\mathrm{d}^{+}$ & - & - & $d^{-}$ & $\mathrm{d}^{-}$ & + \\
\hline 74 & 2-Ketoglutarate $f$ & & & + & + & + & $\mathrm{d}^{+}$ & + & + & + & - & + & + & $d^{+}$ & $\mathrm{d}^{-}$ & + \\
\hline 75 & DL-Norvaline & & & & & + & $\mathrm{d}^{-}$ & + & - & - & $\mathrm{d}^{+}$ & - & - & - & $\mathrm{d}^{-}$ & - \\
\hline 76 & DL-2-Aminobutyrate ${ }^{f}$ & - & & d & - & + & $\mathrm{d}^{+}$ & + & - & - & $\mathrm{d}^{+}$ & $\mathrm{d}^{+}$ & - & - & $\mathrm{d}^{-}$ & - \\
\hline 77 & DL-3-Aminobutyrate $f$ & & & & & + & $\mathrm{d}^{+}$ & + & + & - & - & $\mathrm{d}^{+}$ & - & - & $\mathrm{d}^{-}$ & + \\
\hline 78 & Suberate ${ }^{f}$ & - & & d & + & + & $d^{-}$ & $d^{+}$ & $\mathrm{d}^{+}$ & $d^{+}$ & - & $\mathrm{d}^{+}$ & - & $\mathrm{d}^{+}$ & + & + \\
\hline 79 & L-Citrulline ${ }^{f}$ & & & - & - & + & $\mathrm{d}^{+}$ & $\mathrm{d}^{+}$ & - & - & - & - & - & $d^{-}$ & $\mathrm{d}$ & $d^{-}$ \\
\hline 80 & Amylamine $f^{f}$ & - & & - & + & + & $\mathrm{d}^{+}$ & - & + & - & - & - & - & - & - & - \\
\hline 81 & Arbutinf & & & & & + & $\mathrm{d}^{+}$ & - & - & $\mathrm{d}^{-}$ & + & - & - & - & - & - \\
\hline 82 & Salicin $f$ & & + & d & + & + & $\mathrm{d}^{+}$ & - & - & $d^{-}$ & + & - & - & - & - & - \\
\hline 83 & Pimelate $f^{f}$ & - & - & d & d & + & - & $\mathrm{d}^{+}$ & - & - & - & $\mathrm{d}^{+}$ & - & $\mathrm{d}^{+}$ & + & + \\
\hline 84 & Diaminobutane $^{f}$ & & & & & + & + & - & - & - & - & - & - & - & - & - \\
\hline 85 & L-Rhamnose ${ }^{f}$ & - & - & - & - & - & - & - & + & - & + & $\mathrm{d}^{+}$ & $\mathrm{d}^{+}$ & - & - & - \\
\hline 86 & 5-Ketogluconate ${ }^{f}$ & & & & & + & - & + & + & $\mathrm{d}^{-}$ & + & $\mathrm{d}^{+}$ & - & - & $d^{-}$ & - \\
\hline 87 & Xylitof & & + & & & + & - & $\mathrm{d}^{+}$ & + & - & - & + & - & - & - & - \\
\hline 88 & L-Arabitol $f$ & & & & & + & - & - & + & - & - & + & - & - & - & - \\
\hline 89 & Glycolate $^{f}$ & - & - & - & - & + & - & $d^{-}$ & $d^{-}$ & - & - & - & - & - & + & + \\
\hline 90 & Citraconate $^{f}$ & + & + & - & - & + & - & $\mathrm{d}^{-}$ & - & - & + & - & - & - & + & + \\
\hline 91 & $m$-Hydroxybenzoate $f$ & - & $d^{-}$ & - & - & + & - & $d^{+}$ & - & - & - & - & - & - & $d^{-}$ & - \\
\hline 92 & Butylamine $^{f}$ & & & & & + & - & - & - & - & - & - & - & - & - & - \\
\hline 93 & Tryptamine $^{f}$ & - & - & - & - & + & - & - & - & + & - & - & - & - & - & - \\
\hline 94 & Adonitolf & - & $d^{-}$ & & $\mathrm{d}$ & + & - & + & + & + & - & + & + & - & - & - \\
\hline 95 & Ethylamine & & & - & & - & - & - & - & - & - & - & - & - & - & - \\
\hline 96 & Erythritoff & & - & - & + & - & - & - & - & - & - & - & - & - & - & - \\
\hline 97 & Oxalate ${ }^{f}$ & & - & - & & - & - & - & + & $\mathrm{d}^{-}$ & - & - & - & - & $\mathrm{d}^{-}$ & + \\
\hline 98 & L-Methionine & & - & & - & - & - & - & - & - & - & - & - & - & $d^{-}$ & $d^{-}$ \\
\hline 99 & Maleate & & - & - & - & $d^{-}$ & - & $d^{-}$ & - & - & - & - & - & - & $\mathrm{d}^{-}$ & + \\
\hline 100 & D-Tartrate $f$ & + & + & - & - & - & - & + & - & - & + & - & - & - & $\mathrm{d}^{-}$ & $d^{+}$ \\
\hline 101 & Mesaconate ${ }^{f}$ & + & $\mathrm{d}^{+}$ & - & - & - & - & + & - & - & $d^{-}$ & - & - & - & + & - \\
\hline 102 & Terephthalate & & & - & - & - & - & $d^{+}$ & - & - & - & - & - & - & - & - \\
\hline 103 & Itaconate & - & - & - & - & - & + & - & - & - & - & + & - & - & + & - \\
\hline 104 & Lactose & + & & - & - & - & $\mathrm{d}^{-}$ & - & - & - & - & - & + & - & - & - \\
\hline 105 & D-Xylose ${ }^{f}$ & + & + & + & - & $\mathrm{d}^{+}$ & + & + & + & + & + & $\mathrm{d}^{+}$ & - & - & - & + \\
\hline 106 & L-Xylose & & & & & $d^{-}$ & - & - & - & - & - & - & - & - & - & - \\
\hline 107 & D-Tryptophan & & & - & - & $\mathrm{d}^{-}$ & - & - & - & - & - & - & - & - & - & - \\
\hline 108 & L-Mandelate & - & & - & $\mathrm{d}$ & $d^{+}$ & - & $d^{-}$ & - & - & - & - & - & - & $d^{-}$ & $d^{+}$ \\
\hline 109 & Methyl-D-glucoside & & - & & & $d^{-}$ & - & - & - & - & - & - & - & - & - & - \\
\hline 110 & D-Turanose & & - & & & $d^{-}$ & - & - & - & - & - & - & - & - & - & - \\
\hline 111 & $o$-Hydroxybenzoate ${ }^{f}$ & & & - & - & $d^{+}$ & - & - & - & + & - & - & - & - & $d^{-}$ & - \\
\hline 112 & Glycine $^{f}$ & - & - & + & - & $\mathrm{d}^{-}$ & - & - & - & - & $d^{+}$ & $\mathrm{d}^{+}$ & - & - & - & $d^{+}$ \\
\hline 113 & Urea & & & & & $d^{-}$ & - & - & - & - & - & - & - & - & - & - \\
\hline 114 & Acetamide & & & & & $d^{-}$ & - & - & - & - & - & - & - & - & - & - \\
\hline 115 & D-Melibiose ${ }^{f}$ & & + & & & $\mathrm{d}^{-}$ & - & - & - & + & - & - & - & - & - & - \\
\hline 116 & L-Tartrate ${ }^{f}$ & - & + & - & - & $d^{+}$ & - & + & - & - & + & - & - & $\mathrm{d}^{-}$ & - & + \\
\hline 117 & Benzoate $^{f}$ & - & $\mathrm{d}^{-}$ & + & + & $d^{+}$ & + & + & - & - & - & - & - & $\mathrm{d}^{+}$ & + & $d^{+}$ \\
\hline 118 & D-Cellobiose ${ }^{f}$ & & + & + & + & $\mathrm{d}^{+}$ & + & $\mathrm{d}^{+}$ & $d^{-}$ & $\mathrm{d}^{+}$ & + & - & - & - & - & - \\
\hline 119 & Benzylamine $f$ & & - & - & - & $d^{+}$ & + & $d^{-}$ & $\mathrm{d}^{+}$ & - & - & $d^{-}$ & - & - & - & - \\
\hline 120 & Histamine $^{f}$ & & - & - & - & $\mathrm{d}^{+}$ & + & - & - & - & - & - & - & - & - & - \\
\hline 121 & Isovalerate $f$ & & & & & $d^{+}$ & + & - & $\mathrm{d}^{-}$ & - & - & - & - & $\mathrm{d}^{+}$ & + & + \\
\hline 122 & Sucrose $\mathrm{e}^{f}$ & - & - & + & + & $\mathrm{d}^{+}$ & + & - & + & - & - & + & - & + & - & - \\
\hline
\end{tabular}




\begin{tabular}{|c|c|c|c|c|c|c|c|c|c|c|c|c|c|c|c|c|}
\hline No. & Substrate $^{a}$ & 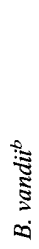 & 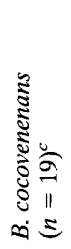 & 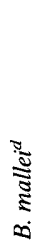 & 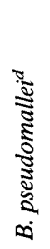 & 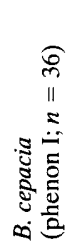 & 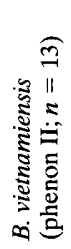 & 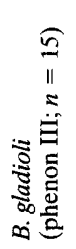 & 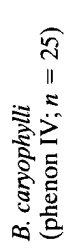 & 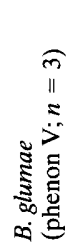 & 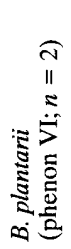 & 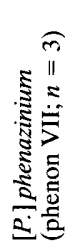 & 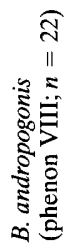 & 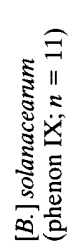 & 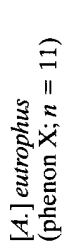 & 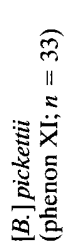 \\
\hline 123 & $\beta$-Gentiobiose ${ }^{f}$ & & & & & $\mathrm{~d}^{+}$ & + & - & $\mathrm{d}^{-}$ & $\mathrm{d}^{-}$ & + & - & - & - & - & - \\
\hline 124 & meso-Tartrate $f$ & & & - & - & $d^{+}$ & $\mathrm{d}^{-}$ & + & + & - & - & + & - & - & $\mathrm{d}^{-}$ & $\mathrm{d}^{+}$ \\
\hline 125 & D-Raffinose ${ }^{f}$ & & - & & & $d^{+}$ & $\mathrm{d}^{+}$ & - & + & $\mathrm{d}^{+}$ & - & - & - & - & - & - \\
\hline 126 & Spermine $\mathrm{e}^{f}$ & & & & & $\mathrm{~d}^{-}$ & $\mathrm{d}^{-}$ & - & - & - & - & - & - & - & - & - \\
\hline 127 & Maltose $\mathrm{e}^{f}$ & & - & d & + & $\mathrm{d}^{-}$ & - & - & - & - & - & - & - & - & - & - \\
\hline 128 & Methyl-D-mannoside & - & & & & $\mathrm{d}^{-}$ & - & - & - & - & - & - & - & - & - & - \\
\hline 129 & D-Melezitose & & & & & - & - & - & - & - & - & - & - & - & - & - \\
\hline 130 & Starch & & - & & & - & - & - & - & - & - & - & - & - & - & - \\
\hline 131 & D-Mandelate & & & & & - & - & - & - & - & - & - & - & - & - & - \\
\hline 132 & Trigonelline & & & $\mathrm{d}$ & $\mathrm{d}$ & - & - & - & - & - & - & - & - & - & - & - \\
\hline 133 & Glycogen & & & & & - & - & - & - & - & - & - & - & - & - & - \\
\hline 134 & Creatine & & - & - & - & - & - & - & - & - & - & - & - & - & - & - \\
\hline 135 & 3-Aminobenzoate & & & - & - & - & - & - & - & - & - & - & - & - & - & - \\
\hline 136 & 4-Aminobenzoate & + & + & - & - & - & - & - & - & - & - & - & - & - & - & - \\
\hline 137 & Phthalate & & & - & - & - & - & - & - & - & - & - & - & - & - & - \\
\hline
\end{tabular}

${ }^{a}$ All of the strains which we tested used the following substrates: gluconate, fumarate, L-malate, L-aspartate, and L-glutamate. None of the strains used inulin, methyl-xyloside, L-sorbose, esculin, and isophthalate.

${ }^{b}$ Data from reference 63

${ }^{c}$ Data from reference $74 . n$ is the number of strains studied.

${ }^{d}$ Data for the type strains of $B$. mallei and $B$. pseudomallei from reference 73 .

${ }^{e}+$, more than $90 \%$ of the strains gave a positive reaction; - , fewer than $10 \%$ of the strains gave a positive reaction, $\mathrm{d}^{+}$, between 10 and $90 \%$ of the strains gave a positive reaction and the type strain was positive; $\mathrm{d}^{-}$, between 10 and $90 \%$ of the strains gave a positive reaction and the type strain was negative.

${ }^{f}$ Characteristics that differentiate members of the genus Burkholderia.

Numerical analysis of auxanographic results. The auxanographic characteristics of the members of the solanacearum rRNA complex are presented in Table 2. Figure 1 shows a dendrogram which was obtained by using unweighted average pair group clustering of $S$ values. All strains clustered above an $S$ value of $64 \%$. The reproducibility of the tests was good; the average $S$ values for 10 strains tested in duplicate (Fig. 1) were between 90 and $95 \%$. Eleven phena (phena I to XI) containing two or more strains were delineated; two strains $(B$. cepacia LMG 6995 and [B.] solanacearum LMG 2295) did not belong to any of these phena. Of the 11 phena which we identified, 10 contain only members of previously described species. Phena I, III, IV, V, VI, VII, IX, and XI contain only B. cepacia, $B$. gladioli, B. caryophylli, B. glumae, B. plantarii, $[P$. $]$ phenazinium, $[B$.$] solanacearum, and [B$.$] pickettii strains, respectively.$ "Pseudomonas oxalaticus" and [A.] eutrophus strains constitute phenon $\mathrm{X}$, while phenon VIII contains only $[P$.] andropogonis and $[P$.$] woodsii strains. The isolates from Vietnam are mem-$ bers of phenon II, which also contains two strains received as B. cepacia LMG 6998 and LMG 6999.

B. cepacia contains plant-pathogenic organisms, saprophytic bacteria, and some clinical isolates. Despite the internal heterogeneity of this species, all $B$. cepacia strains except the two phenon II strains and strain LMG 6995 are phenotypically identical. One of our isolates, strain PHP7, which was isolated from a maize rhizosphere, also belongs to this cluster (phenon I). The description of $B$. cepacia deduced from the auxanographic phenon I data corresponds well to the description given by Palleroni and Holmes (45). Our auxanographic results for phenon I differ in at least 17 carbon sources from the results that Yabuuchi et al. (73) obtained for the type strain of B. cepacia but correspond better to the results of Palleroni (43) and to the results that Urakami et al. obtained for 45 substrates (63). Our results and those of Palleroni (43) differ for only seven carbon sources (ethylamine [substrate 95], L-norleucine [substrate 48], L-rhamnose [substrate 85], L-valine [substrate 23], maltose [substrate 127], DL-2-aminobutyrate [substrate 76], and glycine [substrate 112]), and our results and the results of Urakami et al. (63) differ only for DL-4-aminobutyrate (substrate 20). Although a clear-cut distinction between possible ecological groups could not be made, it is remarkable that all of the strains isolated from Allium cepa grew on L-arabinose (substrate 32), D-xylose (substrate 105), sucrose (substrate 122), L-tartrate (substrate 116), and $o$-hydroxybenzoate (substrate 111) but not on maltose (substrate 127) and D-turanose (substrate 110), while this combination of characteristics was never found for a clinical isolate.

The two aberrant $B$. cepacia strains belonging to phenon II were of clinical origin; these strains constitute a phenon together with the $\mathrm{N}_{2}$-fixing isolates from Vietnam. Within this phenon the TVV strains are more similar to each other than they are to the two aberrant $B$. cepacia strains.

The phenon III $B$. gladioli strains, including the type strain, form a very homogeneous group (mean $S$ value, 91.0\%) and correspond phenotypically to the species $B$. gladioli as described by Ballard et al. (4), although there is an important difference in two characteristics, growth on sucrose (substrate 122) and growth on trigonelline (substrate 132); these two characteristics were found to be negative in this study and also were found to be negative for the reference strain by Yabuuchi et al. (73) and for the type strain by Urakami et al. (63). We observed that the type strain grew slightly on D-cellobiose (substrate 118), while Yabuuchi et al. (73) reported negative results for this test. Growth on D-cellobiose (substrate 118) is impor- 
Cluster no. Taxon name

I B. cepacia

II B. vietnamiensis

III

B. gladioli

IV

B. caryophylli

V
B. glumae

VI

B. plantarii

VII

[P.] phenazinium

VIII [B.] andropogonis

IX

[B.] solanacearum

XI

[B.] pickettii

X [A.] eutrophus and

"P.oxalaticus"

100

90

80

70

\begin{tabular}{|ll|}
\hline LMG1222T & LMG1222BT \\
LMG6864t1 & LMG6887 \\
LMG6959 & LMG6961t1 \\
LMG6992 & LMG696011 \\
LMG6032 & LMG6862t1 \\
LMG1275 & LMG6889t1 \\
LMG6980 & LMG6865 \\
LMG6981t3 & LMG6981t1 \\
LMG6989t1 & LMG6981t2 \\
LMG6996 & LMG6986 \\
LMG6987t1 & LMG6859 \\
LMG6861t1 & LMG6988 \\
LMG6993 & LMG686311 \\
LMG6991t1 & LMG6992 \\
LMG6990t1 & LMG6994 \\
LMG6997 & LMG7000 \\
LMG6860t1 & PHP7 \\
LMG6963t1 & LMG6964 \\
LMG6888 & \\
\hline
\end{tabular}

LMG6998 LMG6999

$\begin{array}{ll}\text { TVV72 } & \text { TVV74 } \\ \text { TVV127 } & \text { TVV135 }\end{array}$

$\begin{array}{ll}\text { TVV127 } & \text { TVV135 } \\ \text { TVV116 } & \text { TVV128 }\end{array}$

TVV75T TVV69

TVV1 15

TVVi15

LMG2121 LMG2121B

LMG6954 LMG6955

LMG6881 LMG6882

LMC6880t1 LMG6957

LMG6915 LMG6979

LMG6958 LMG6884

LMG2216T LMG2216BT

LMG6878

\begin{tabular}{|ll|}
\hline LMG2157 & LMG2155T \\
LMG2156 & LMG2155BT \\
\hline
\end{tabular}

\begin{tabular}{ll} 
LMG2156 & LMG2155BT \\
LMG6876 & LMG6978 \\
\hline
\end{tabular}

\begin{tabular}{|ll|}
\hline LMG1277 & LMG2196T \\
LMG21961 & LMG6952 \\
\hline
\end{tabular}

LMG9035T LMG10908

LMG2247T LMG6868t1 LMG6868t2

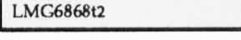

\begin{tabular}{|ll|}
\hline LMG2126 & LMG6947 \\
LMG2363 & LMG2362B \\
LMG2127 & LMG1279 \\
LMG2128 & LMG2362 \\
LMG2129T & LMG6943 \\
LMG6944 & LMG2227 \\
LMG2129BT & LMG2329 \\
LMG6946 & LMG6872 \\
LMG6875 & LMG6948 \\
LMG6949 & LMG6950 \\
LMG6873 & LMG6945 \\
LMG6874 & LMG6942 \\
\hline & \\
\hline LMG2299T & LMG2299BT \\
LMG2296 & LMG2301 \\
LMG2303 & LMG2298 \\
LMG2300 & LMG2305 \\
LMG2306 & LMG2297 \\
LMG2304 & LMG5839 \\
\hline
\end{tabular}

$\begin{array}{ll}\text { LMG2306 } & \text { LMG2297 } \\ \text { LMG5839 }\end{array}$

LMG2295

LMG6995

$\begin{array}{ll}\text { LMG2233 } & \text { LMG2236 } \\ \text { LMG2234t1 } & \text { LMG2235** }\end{array}$

LMG1199T LMG1200

LMG1190 LMG1210

LMG1211 LMG1206

LMG5942T LMG7145

LMG5942BT LMG7002

LMG7003 LMG7004

LMG7058

LMG7057

LMG7001

MG7008 LMG7159

MG7140 LMG7160

LMG7143 LMG7012

MG7014 LMG7017

MG7009 LMG7013

LMG7147t1 LMG6871

LMG7141 LMG7018
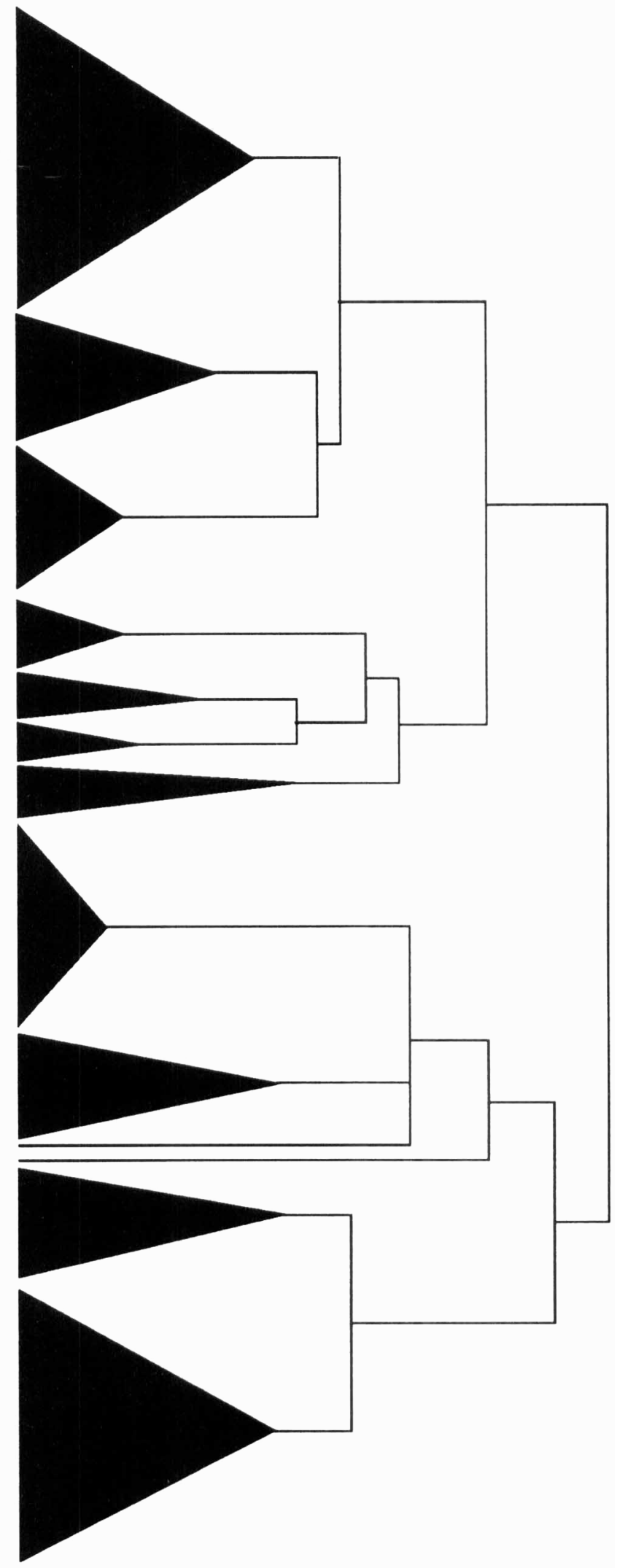

FIG. 1. Dendrogram obtained from an unweighted average pair group cluster analysis of similarity coefficients of strains belonging to the solanacearum rRNA complex. The results of 137 auxanographic tests were compared in this analysis. The suffix B indicates that a strain was tested twice. B., Burkholderia; A., Alcaligenes; P., Pseudomonas. 
tant since this characteristic has been used as a diagnostic characteristic for the nonfluorescent phytopathogenic [Pseudomonas] species. Most of our results correspond to the results of Urakami et al. (63); the exceptions are the results for 4-aminobenzoate (substrate 136) and DL-2-aminobutyrate (substrate 76). We could not detect auxanographic differences between the two pathovars of $B$. gladioli; the only difference which we could confirm was darkening of the culture medium by some strains of $B$. gladioli pv. alliicola but not by any member of $B$. gladioli pv. gladioli.

Phenon IV corresponds to $B$. caryophylli, although the phenotypic description of Ballard et al. (4) and the results obtained for the type strain by Yabuuchi et al. (73) differ from our data, especially the amino acid data (we found more positive reactions). In addition, differences in growth on salicin (substrate 82), adonitol (substrate 94), malonate (substrate 73), azelate (substrate 61), sebacate (substrate 62), sarcosine (substrate 44), benzylamine (substrate 119), D-cellobiose (substrate 118), $n$-valerate (substrate 46), phenylacetate (substrate 64), oxalate (substrate 97), and amylamine (substrate 80 ) were found. The five strains included in this phenon exhibit a high level of phenotypic similarity $(92.3 \%)$, although they have different geographic origins. Most of results are consistent with those of Urakami et al. (63); the exceptions are the data for growth on L-threonine (substrate 13), azelate (substrate 61), L-rhamnose (substrate 85), and adonitol (substrate 94).

All three strains of $B$. glumae that we investigated fell into phenon V, although strain LMG 1277 was somewhat different from the two other strains. The description of this species given by Kurita and Tabei (36) was based mainly on acidification of sugars; consequently, we could not compare this description with most of our auxanographic results, which correspond to the data of Azegami et al. (2) and Urakami et al. (63); the only exceptions were our data for growth on L-threonine (substrate 13), caprylate (substrate 53), levulinate (substrate 54), and adonitol (substrate 94). Some characteristic features of $B$. glumae deduced from our auxanographic data were growth on adonitol (substrate 94), D-xylose (substrate 105), L-fucose (substrate 69), dulcitol (substrate 65), $p$-hydroxybenzoate (substrate 19), and tryptamine (substrate 93) but no growth on citraconate (substrate 90), DL-3-aminobutyrate (substrate 77), meso-tartrate (substrate 124), and benzoate (substrate 117).

Azegami et al. (2) described [P.] plantarii, which was later transferred to the genus Burkholderia as $B$. plantarii (63). We investigated two representative strains of $B$. plantarii, including the type strain; these organisms constitute a separate phenon (phenon VI) that is most similar to B. glumae. These taxa differ from each other in the following characteristics: growth on adonitol (substrate 94), citraconate (substrate 90), D-tartrate (substrate 100), L-rhamnose (substrate 85), L-tartrate (substrate 116), tryptamine (substrate 93), D-melibiose (substrate 115), $o$-hydroxybenzoate (substrate 111), L-tryptophan (substrate 43), 2-aminobenzoate (substrate 59), and 2-ketoglutarate (substrate 74). Again, most of the results obtained with 45 substrates by Urakami et al. (63) were identical to our results; the only exceptions were the results for growth on D-tartrate (substrate 100), glycine (substrate 112), and sucrose (substrate 122).

Three strains of $[P$.$] phenazinium constitute phenon VII;$ although these strains have a similarity value of only approximately $80 \%$, our results confirmed the description given by Bell and Turner (6).

We could not detect any phenotypic differences between the members of phenon VIII ([P.] woodsii and $[P$.$] andropogonis);$ these organisms are almost identical phenotypically and form a very tight cluster ( $S$ value, $94.3 \%$ ). Our results are consistent with the description of Goto and Starr (27) for [P.] andropogonis and with the description of Burkholder and Guterman (14) for $[P$.$] woodsii. The latter species was originally described as$ pathogenic for Dianthus caryophyllus, while $[P$.$] andropogonis$ causes symptoms in at least 12 plant species belonging to 10 genera $(1,13,15,27,30,51)$. Although the symptoms can vary between different plants, the names $[P$.$] woodsii and [P$.$] an-$ dropogonis can be considered synonyms because of the nearly identical phenotypic profiles of these organisms.

Our results confirmed the description of [B.] solanacearum (phenon IX), a taxon that has been studied intensively previously $(11,29,44)$. However, we observed large variations in growth for the following substrates: acetate (substrate 7), benzoate (substrate 117), isobutyrate (substrate 34), suberate (substrate 78), 2-ketoglutarate (substrate 74), D- $\alpha$-alanine (substrate 8), L-leucine (substrate 42), and L-tryptophan (substrate 43). Also, the subdivision of this species into four biovars (29) was confirmed, and our results represent a completion of Hayward's study (29). Our results differ from the results that Urakami et al. (63) obtained for the type strain of [B.] solanacearum in only a few characteristics (growth on D-galactose [substrate 9], $n$-caproate [substrate 51], heptanoate [substrate 52], and benzoate [substrate 117]).

We found that four strains of "P. oxalaticus" (34) and seven strains of [A.] eutrophus are members of phenon $\mathrm{X}$, which confirmed the auxanographic results of Jenni et al. (32). Within this phenon the co-type strain of " $P$. oxalaticus" differs most from the three other "P. oxalaticus" strains and from all of the $[A$. $]$ eutrophus strains.

Phenon XI contains only [B.] pickettii strains; in general, our results are consistent with the descriptions given by Ralston et al. (48) and Palleroni (43) but differ from some of the results obtained by Yabuuchi et al. (73) and Urakami et al. (63) for the type strain. The core of this cluster ( 28 strains) is very homogeneous (mean $S$ value, $89.8 \%$ ). All of the auxanographic features that differentiate the phena are shown in Table 2; for comparison, the results obtained with the type strains of $B$. mallei (73), Burkholderia pseudomallei (73), [P.] cocovenenans (74), and $B$. vandii (63) are included in Table 2.

Nitrogenase activity. All of the B. vietnamiensis TVV strains isolated from the rhizosphere of rice plants were positive for nitrogenase activity (Table 3). Only two of the Burkholderia reference strains tested in this study (LMG 6998 and LMG 6999) exhibited nitrogenase activity. These strains, together with the TVV strains, constitute phenon II (Fig. 1) and produce ornibactin siderophores (41).

DNA-rRNA hybridization. Previous hybridization results allowed us to define the solanacearum rRNA complex $(22,23)$ and to show that $[A$. $]$ eutrophus is the only group belonging to this complex that does not belong to one of the sections of the genus Pseudomonas as defined by Palleroni (43). Hybridization experiments performed with rRNA from [A.] eutrophus LMG $1199^{\mathrm{T}}$ clearly revealed that this species constitutes a separate genotypic cluster $\left[T_{m(e)}, 78.2\right.$ to $\left.80.0^{\circ} \mathrm{C}\right]$ on a separate rRNA branch (Fig. 2 and Table 4 ) that splits off at a $\Delta T_{m(e)}$ value of $7^{\circ} \mathrm{C}$. The results of preliminary hybridizations between DNAs from two representative TVV rice isolates and $[B$.$] solanacea-$ rum LMG $2299^{\mathrm{T}}$ rRNA demonstrated that these rice isolates also belong to the solanacearum rRNA complex. To determine the genotypic relationships among the members of this rRNA complex, we concentrated on the phena that were most closely related to $B$. cepacia. We prepared a $23 \mathrm{~S}$ rRNA probe from $B$. cepacia LMG $1222^{\mathrm{T}}$ and hybridized it with DNAs from representative strains of the different phena. The results of the DNA-rRNA hybridization experiments are shown in Table 4. Members of phena I and II had very high $T_{m(e)}$ values (79.6 to 
TABLE 3. Nitrogenase activities of reference strains of Burkholderia species

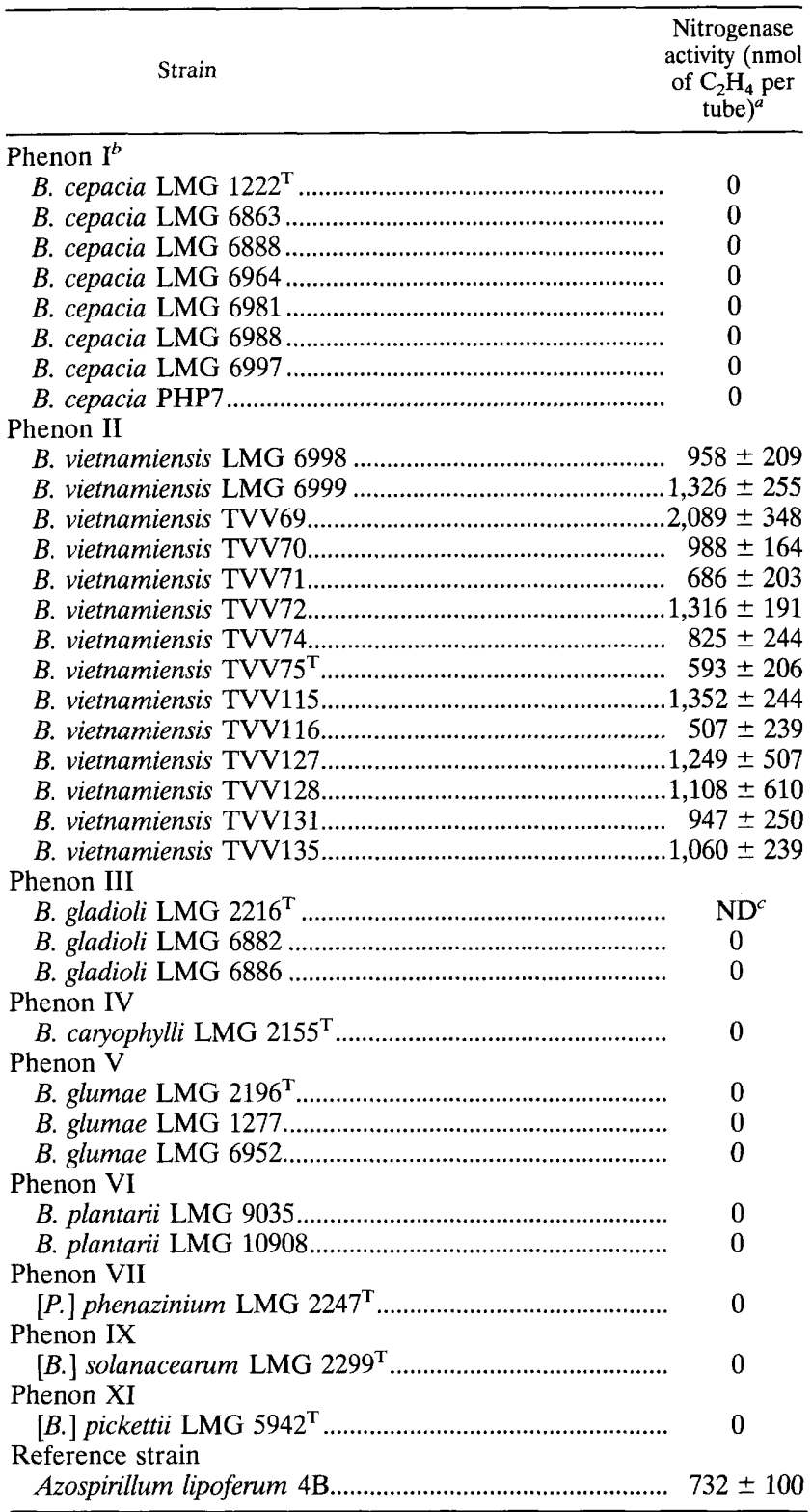

${ }^{a}$ Averages \pm standard deviations of the values from three experiments.

${ }^{b}$ See Fig. 1 .

${ }^{c} \mathrm{ND}$, not determined.

$81.1^{\circ} \mathrm{C}$ ), indicating that these two clusters are genotypically very similar. Of all of the other representative strains of the different phena examined, only $B$. gladioli LMG 6882 (phenon III) and the type strains of $B$. plantarii (phenon VI), $[P$.$] co-$ covenenans, and $B$. glumae (phenon $\mathrm{V}$ ) had $T_{m(e)}$ values that were significantly higher $\left(78.4\right.$ to $\left.79.1^{\circ} \mathrm{C}\right)$ than the $T_{m(e)}$ values of the representatives of the other phena $\left(75.2\right.$ to $\left.76.7^{\circ} \mathrm{C}\right) . \mathrm{T}$. mixta LMG $11547^{\mathrm{T}}$ does indeed not belong in this rRNA complex since its DNA-rRNA hybrid with rRNA from $B$. $c e$ pacia LMG $1222^{\mathrm{T}}$ had a $T_{m(e)}$ of about $70^{\circ} \mathrm{C}$.

DNA-DNA hybridization. The unstandardized reassociation values for homoduplexes ranged from 69 to $100 \%$. The levels of reassociation in control tubes containing only labeled DNA

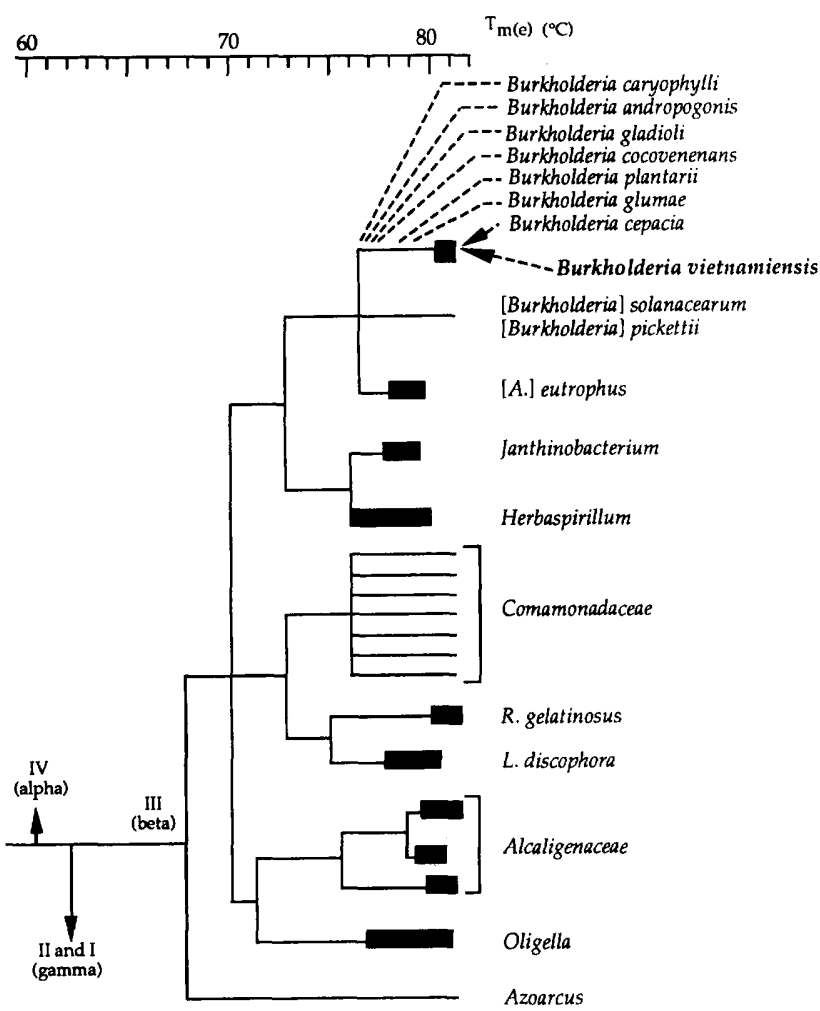

FIG. 2. $T_{m(e)}$ dendrogram showing the position of the Burkholderia rRNA branch within rRNA superfamily III (beta subclass). A., Alcaligenes; R., Rubrivivax; L., Leptothrix.

ranged from 5 to $13 \%$. The $T_{m}$ values for homoduplexes (in $0.42 \mathrm{M} \mathrm{NaCl}$ ) ranged from 95 to $97.5^{\circ} \mathrm{C}$. The levels of relative DNA homology at $70^{\circ} \mathrm{C}$ are shown in Table 5 .

The two reference B. cepacia strains, LMG $1222^{\mathrm{T}}$ and LMG 6964, which belong to different subclusters of phenon I, gave comparable results with all of the strains studied. Of the nine collection strains previously identified as $B$. cepacia, seven strains belonging to phenon I exhibited high levels of homology (up to 65\%) with B. cepacia LMG 6964 and thus belong to this species. The two remaining strains (LMG 6998 and LMG 6999) and the 12 strains isolated from the rice rhizosphere constitute another DNA homology group. Within this group, which corresponds to phenon II (Fig. 1), the strains exhibited levels of homology of more than $85 \%$, and they exhibited levels of homology of less than $50 \%$ and $\Delta T_{m}$ values ranging from 5 to $6^{\circ} \mathrm{C}$ with $B$. cepacia strains (members of phenon 1 ). The members of both of these DNA homology groups exhibited 5 to $26 \%$ DNA relatedness with members of the other clusters belonging to this RNA complex and less than 5\% DNA relatedness with other pseudomonad reference strains.

\section{DISCUSSION}

On the basis of the results of our DNA-rRNA hybridization experiments we concluded that the rRNA complex which we studied contains at least three separate rRNA branches (Fig. 2 ), namely, the $[B$.] solanacearum rRNA branch, the $B$. cepacia rRNA branch, and the [A.] eutrophus rRNA branch. Recent analyses of about 80 and 89 to $97 \%$ of relevant $16 \mathrm{~S}$ ribosomal DNA sequences provided more details concerning the phylo- 
TABLE 4. Results of the DNA-rRNA hybridization experiments performed with rRNAs from B. cepacia LMG $1222^{\mathrm{T}}$ and $[A$.] eutrophus LMG $1199^{\mathrm{T}}$ and DNAs from representatives of the different phena

\begin{tabular}{|c|c|c|}
\hline \multirow{2}{*}{ Source of DNA } & \multicolumn{2}{|c|}{$\begin{array}{c}T_{m(e)}\left({ }^{\circ} \mathrm{C}\right) \text { of hybrid with } \\
\text { rRNA from: }\end{array}$} \\
\hline & $\begin{array}{l}\text { B. cepacia } \\
\text { LMG } 1222^{\mathrm{T}}\end{array}$ & $\begin{array}{l}\text { [A.] eutrophus } \\
\text { LMG } 1199^{\mathrm{T}}\end{array}$ \\
\hline \multicolumn{3}{|l|}{ Phenon $I^{a}$} \\
\hline B. cepacia LMG $1222^{\mathrm{T}}$ & 81.1 & 75.0 \\
\hline B. cepacia LMG 6998 & 80.2 & \\
\hline B. cepacia LMG 6883 & 80.4 & \\
\hline B. cepacia LMG 6993 & 81.1 & \\
\hline B. cepacia $\mathrm{LMG} 6888$ & 81.1 & \\
\hline B. cepacia LMG 6981 & 80.6 & \\
\hline B. cepacia LMG 6988 & 79.6 & \\
\hline \multicolumn{3}{|l|}{ Phenon II } \\
\hline B. vietnamiensis TVV116 & 80.5 & \\
\hline B. vietnamiensis TVV69 & 80.9 & \\
\hline B. vietnamiensis $\mathrm{TVV} 75^{\mathrm{T}}$ & 81.0 & \\
\hline B. vietnamiensis TVV70 & 80.4 & \\
\hline B. vietnamiensis TVV131 & 80.1 & \\
\hline \multicolumn{3}{|l|}{ Phenon III } \\
\hline B. gladioli LMG 6882 & 78.6 & \\
\hline B. gladioli LMG $2216^{\mathrm{T}}$ & 75.0 & \\
\hline \multicolumn{3}{|l|}{ Phenon IV } \\
\hline B. caryophylli $\mathrm{LMG} 2299^{\mathrm{T}}$ & 75.5 & 75.0 \\
\hline \multicolumn{3}{|l|}{ Phenon V } \\
\hline B. glumae LMG $2196^{\mathrm{T}}$ & 79.1 & \\
\hline \multicolumn{3}{|l|}{ Phenon VI } \\
\hline B. plantarii LMG $9035^{\mathrm{T}}$ & 78.4 & \\
\hline \multicolumn{3}{|l|}{ Phenon VII } \\
\hline$[P$.$] phenazinium LMG 2247^{\mathrm{T}}$ & & 74.5 \\
\hline \multicolumn{3}{|l|}{ Phenon VIII } \\
\hline B. andropogonis LMG $2729^{\mathrm{T}}$ & 76.7 & 73.5 \\
\hline B. woodsii LMG $2362^{\mathrm{T}}$ & & 74.0 \\
\hline \multicolumn{3}{|l|}{ Phenon IX } \\
\hline$\left[\right.$ B.] solanacearum LMG $2299^{\mathrm{T}}$ & 75.2 & 75.5 \\
\hline [B.] solanacearum LMG 2297 & & 75.5 \\
\hline \multicolumn{3}{|l|}{ Other $[B$.$] solanacearum strains$} \\
\hline [B.] solanacearum LMG 2291 & & 76.5 \\
\hline [B.] solanacearum LMG 2293 & & 76.0 \\
\hline \multicolumn{3}{|l|}{ Phenon X } \\
\hline "P. oxalaticus" LMG 2235 & & 76.0 \\
\hline [A.] eutrophus LMG $1199^{\mathrm{T}}$ & & 79.5 \\
\hline [A.] eutrophus LMG 1200 & & 79.0 \\
\hline [A.] eutrophus LMG 1201 & & 79.5 \\
\hline [A.] eutrophus LMG 1206 & & 79.5 \\
\hline [A.] eutrophus LMG 1207 & & 78.2 \\
\hline [A.] eutrophus LMG 1209 & & 79.5 \\
\hline \multicolumn{3}{|l|}{ Other $[A$.$] eutrophus strains$} \\
\hline [A.] eutrophus LMG 1202 & & 78.5 \\
\hline [A.] eutrophus LMG 1203 & & 79.5 \\
\hline [A.] eutrophus LMG 1204 & & 80.0 \\
\hline [A.] eutrophus LMG 1205 & & 80.0 \\
\hline \multicolumn{3}{|l|}{ Phenon XI } \\
\hline [B.] pickettii ATCC 27511 & 75.3 & \\
\hline \multicolumn{3}{|l|}{ Other strains } \\
\hline B. cocovenenans LMG $11626^{\mathrm{T}}$ & 77.7 & \\
\hline$T$. mixta LMG $11547^{\mathrm{T}}$ & 69.6 & \\
\hline
\end{tabular}

${ }^{a}$ See Fig. 1.

genetic relationships among the members of the solanacearum rRNA complex $(38,73)$. It was shown that $B$. cepacia is closely related to $B$. gladioli and that $[B$.] solanacearum is closely related to $[B$.$] pickettii (38,73)$. Both groups constitute separate lineages. $B$. caryophylli can be considered a regular member of the $B$. cepacia-B. gladioli lineage (38), while $[P$.$] andro-$ pogonis represents a somewhat more removed branch of this lineage. On the other hand, $[A$. $]$ eutrophus is a further removed branch of the $[B$.] solanacearum- $[B$. $]$ pickettii lineage (38). It is our experience that members of an rRNA branch that have $\Delta T_{m(e)}$ values of 4 to $5^{\circ} \mathrm{C}(18)$ can be considered members of a separate genus. Consequently, the $B$. cepacia rRNA branch, which contains, in addition to $B$. cepacia strains, strains representing auxanographic phena II (the $\mathrm{N}_{2}$-fixing strains), III ( $B$. gladioli), V (B. glumae), VI (B. plantarii) (Table 4), and $[P$. cocovenenans, constitutes at least a separate genus. The ribosomal DNA sequencing results of Li et al. (38) clearly revealed that $B$. caryophylli (phenon IV) and $[P$.] andropogonis (phenon VIII) can be considered members of the same genus, as mentioned above. It is our strategy to propose generic status only when there are phenotypic and/or chemotaxonomic parameters available to describe and differentiate the genus. From the assimilation results it is evident that phena I to VI, phenon VIII, [P.] cocovenenans, B. mallei, B. pseudomallei, and B. vandii do share characteristics that can differentiate them as a taxonomic entity from the other phena representing the other lineage. Fatty acid profiles also differentiate the proposed new genus from the $[B$.$] solanacearum- [B$.$] pickettii lineage since it$ has been shown that $B$. cepacia, $B$. caryophylli, $B$. gladioli, $B$. glumae, $B$. plantarii, [P.] andropogonis, $B$. mallei, and $B$. pseudomallei constitute a separate fatty acid cluster $(31,59,63$, 73). This fatty acid cluster is characterized by a relatively high percentage of the 3-hydroxy fatty acid 3-OH $\mathrm{C}_{16: 0}(17$ to $38 \%$ of the total 3-hydroxy fatty acids), while this fatty acid is absent or present in small amounts in members of the $[B$.$] solanacea-$ rum-[B.] pickettii lineage. 3-Hydroxy fatty acids are also present in the members of auxanographic phenon II and in $[P$.] cocovenenans (64). Because the recently proposed species $B$. vandii (63) has the same physiological and chemotaxonomic characteristics, it is also considered a member of this genus.

In summary, we conclude that (i) the members of phena I to VI and phenon VIII, $[P$.$] cocovenenans, B$. vandii, $B$. mallei, and $B$. pseudomalle $i$ constitute a separate genus, for which the name Burkholderia has to be retained because this taxon contains the type species, $B$. cepacia, and (ii) members of the other phenotypic groups and/or other rRNA lineages have to be removed from this genus. These organisms are $[B$.$] solanacea-$ rum, $[B$.$] pickettii, and [A$. ] eutrophus. As no data on the rRNA of $[P$.$] phenazinium are available, we cannot decide whether$ this species also belongs in the emended genus Burkholderia. Within the genus Burkholderia as defined here, the intrageneric relationships were determined by performing DNADNA hybridization studies. No significant levels of DNA-DNA binding were observed between members of the clusters belonging to the $B$. cepacia rRNA branch (Fig. 2) except between members of phenon I (B. cepacia) and members of phenon II (the $\mathrm{N}_{2}$-fixing organisms from Vietnam and two $B$. cepacia strains of clinical origin) (Tables 1 and 2). In addition, the $\Delta T_{m(e)}$ values revealed that the members of the phenotypic clusters designated phena I and II were closely related. However, the levels of DNA relatedness (less than 50\% homology as determined by the $\mathbf{S} 1$ nuclease-trichloroacetic acid method) were not high enough to place these organisms in one species. Within phenon II high levels of DNA-DNA hybridization were found, leading to the conclusion that strains belonging to phenon II constitute a new species. We propose that this new species should be named Burkholderia vietnamiensis because the majority of the strains were isolated in Vietnam. On the basis of other DNA-DNA hybridization results (Table 5) and the DNA-rRNA hybridization results (Table 4), we concluded that the following species should be retained as separate taxa: B. gladioli (phenon III), B. caryophylli (phenon IV), B. glumae 
TABLE 5. Results of DNA-DNA hybridization experiments

\begin{tabular}{|c|c|c|c|c|}
\hline \multirow[b]{2}{*}{ Source of unlabeled DNA } & \multicolumn{4}{|c|}{$\%$ Reassociation at $70^{\circ} \mathrm{C}$ with labeled DNA from: } \\
\hline & $\begin{array}{l}\text { B. cepacia } \\
\text { LMG } 1222^{\mathrm{T}}\end{array}$ & $\begin{array}{l}\text { B. cepacia } \\
\text { LMG } 6964\end{array}$ & $\begin{array}{l}\text { B. vietnamiensis } \\
\text { LMG } 6998\end{array}$ & $\begin{array}{l}\text { B. vietnamiensis } \\
\text { TVV70 }\end{array}$ \\
\hline \multicolumn{5}{|l|}{ Phenon I ${ }^{a}$} \\
\hline $\begin{array}{l}\text { Burkholderia cepacia LMG } 1222^{\mathrm{T}} \\
\text { Burkholderia cepacia LMG } 6964\end{array}$ & 100 & 80 & $\begin{array}{l}40(5.0)^{b} \\
50\end{array}$ & 41 \\
\hline Burkholderia cepacia LMG 6963 & & 71 & & 42 \\
\hline Burkholderia cepacia LMG 6888 & & 77 & 40 & \\
\hline Burkholderia cepacia LMG 6981 & & 71 & $47(5.5)$ & \\
\hline Burkholderia cepacia LMG 6988 & & 68 & $40(5.0)$ & \\
\hline Burkholderia cepacia LMG 6997 & & 65 & $35(6.0)$ & \\
\hline \multicolumn{5}{|l|}{ Phenon II } \\
\hline Burkholderia vietnamiensis LMG 6998 & & $48(5.0)$ & 100 & \\
\hline Burkholderia vietnamiensis LMG 6999 & & $46(5.0)$ & 100 & \\
\hline Burkholderia vietnamiensis TVV70 & 35 & $46(6.0)$ & 88 & 100 \\
\hline Burkholderia vietnamiensis TVV69 & 38 & 47 & 92 & 95 \\
\hline Burkholderia vietnamiensis TVV71 & 35 & 47 & & 93 \\
\hline Burkholderia vietnamiensis TVV74 & 33 & & 85 & 94 \\
\hline Burkholderia vietnamiensis TVV72 & 32 & & & 98 \\
\hline Burkholderia vietnamiensis TVV75? & 34 & & & 102 \\
\hline Burkholderia vietnamiensis TVV115 & 31 & & & 103 \\
\hline Burkholderia vietnamiensis TVV116 & 34 & & & 99 \\
\hline Burkholderia vietnamiensis TVV127 & 32 & & & 95 \\
\hline Burkholderia vietnamiensis TVV128 & 28 & & & 108 \\
\hline Burkholderia vietnamiensis TVV131 & 36 & & & 99 \\
\hline Burkholderia vietnamiensis TVV135 & 36 & & & 101 \\
\hline \multicolumn{5}{|l|}{ Phenon III } \\
\hline Burkholderia gladioli LMG $2216^{\mathrm{T}}$ & & & & 21 \\
\hline Burkholderia gladioli LMG 6882 & & 26 & 22.2 & \\
\hline \multicolumn{5}{|l|}{ Phenon IV } \\
\hline Burkholderia caryophylli LMG $2155^{\mathrm{T}}$ & & 10 & 8 & 12 \\
\hline \multicolumn{5}{|l|}{ Phenon V } \\
\hline Burkholderia glumae LMG $2196^{\mathrm{T}}$ & & & 20 & \\
\hline \multicolumn{5}{|l|}{ Phenon VII } \\
\hline [Pseudomonas] phenazinium LMG $2247^{\mathrm{T}}$ & & 8 & 5 & \\
\hline \multicolumn{5}{|l|}{ Phenon IX } \\
\hline [Burkholderia] solanacearum LMG $2299^{\mathrm{T}}$ & 24 & 5 & & \\
\hline \multicolumn{5}{|l|}{ Phenon XI } \\
\hline [Burkholderia] pickettii LMG $5942^{\mathrm{T}}$ & 9 & 19 & 10 & 16 \\
\hline \multicolumn{5}{|l|}{ Reference strains } \\
\hline Brevundimonas diminuta LMG $1793^{\mathrm{T}}$ & 0 & & & 0 \\
\hline Pseudomonas aeruginosa LMG 6395 & 0 & & & 1 \\
\hline Pseudomonas stutzeri LMG $2333^{\mathrm{T}}$ & & & & 1 \\
\hline Pseudomonas putida LMG $2257^{\mathrm{T}}$ & & & & 1 \\
\hline Sphingomonas paucimobilis $\mathrm{LMG} 1227^{\mathrm{T}}$ & 0 & & & 5 \\
\hline
\end{tabular}

${ }^{a}$ See Fig. 1

${ }^{b}$ The values in parentheses are $\Delta T_{m(e)}$ values (in degrees Celsius).

(phenon V), B. plantarii (phenon VI), and B. andropogonis (phenon VIII). The names B. woodsii and B. andropogonis are probably synonyms. Although genotypic evidence concerning this possible synonymy is not yet available, we propose that these two species should be placed in one species, for which the name Burkholderia andropogonis is proposed on the basis of Rule 42 of the International Code of Nomenclature of Bacteria (37).

From the rRNA and DNA-DNA results of Yabuuchi et al. (73) it is evident that $B$. malle $i$ and $B$. pseudomallei belong to a single genospecies, but it has been proposed that these two species should be kept separate for epidemiological and zoonotic reasons. $B$. cocovenenans has been described as exhibiting $60 \%$ DNA-DNA binding with $B$. cepacia ATCC 13945; our DNA-rRNA hybridization results showed that $B$. cocovenenans is genotypically less similar to $B$. cepacia than $B$. vietnamiensis. DNA-DNA hybridization data (64) confirmed the separate status of $B$. cocovenenans. For $B$. vandii (63) the DNA-DNA homology values obtained with $B$. glumae and $B$. plantarii were at the borderline of species separation, and the final conclusion was based on differences in utilization of carbon compounds. The differentiating auxanographic characteristics of all of the species belonging to the redefined genus Burkholderia are shown in Table 2. Our results corresponded rather well to those obtained for a few strains of each species (including the type strain) by Urakami et al. (63), who used 45 common substrates in their analysis. In the proposed emended description of the genus Burkholderia below we omit characteristics for which different results were obtained by us and Urakami et al. (63).

Emended description of the genus Burkholderia. Burkholderia (Yabuuchi, Kosako, Oyaio, Yano, and Ezaki 1992) (Burk. hold.er'i.a. M.L. dim. ending -ia; M.L. fem. n. Burkholderia, named after W. H. Burkholder, the American bacteriologist 
TABLE 6. Characteristics that differentiate B. cepacia, B. gladioli, and $B$. vietnamiensis

\begin{tabular}{lccc}
\hline \multicolumn{1}{c}{ Characteristic } & B. cepacia & B. vietnamiensis & B. gladioli \\
\hline Nitrogenase activity & - & + & - \\
Denitrification & - & + & - \\
Carbon sources used for growth & & & \\
$\quad$ L-Arabitol & + & - & - \\
Butylamine & + & - & - \\
Tryptamine & + & - & - \\
Adonitol & + & - & + \\
Diaminobutane & + & + & - \\
5-Ketogluconate & + & - & + \\
D-Tartrate & - & - & + \\
Mesaconate & - & - & + \\
Itaconate & - & + & - \\
Siderophore production & & & \\
Cepabactin & + & - & ND \\
Pyochelin & + & - & ND \\
Ornibactin & + & + & ND \\
\hline
\end{tabular}

${ }^{a} \mathrm{ND}$, not determined.

who first discovered the etiological agent of sour skin of onions). Burkholderia cells are gram-negative, nonfermentative, straight rods that have a single polar flagellum or a tuft of polar flagella. A single species, B. mallei, is atrichous and nonmotile. Catalase is produced, and oxidase activity varies between species. The cellular fatty acids are characterized by the presence of 3-hydroxy $\mathrm{C}_{16: 0}$. The type strains of several species are characterized by the presence of two types of ornithine lipids. Most species grow at $40^{\circ} \mathrm{C}$. All species can grow with the following substrates as sole carbon sources: glucose, glycerol, inositol, galactose, sorbitol, and mannitol. Some species are pathogenic for humans, animals, or plants. Isolated from plant material, soil, or clinical samples. Can be recognized on the basis of $16 \mathrm{~S}$ rRNA characteristics (sequence and/or DNArRNA hybridization data). Most strains accumulate polyhydroxybutyrate as carbon reserve material and are capable of ortho cleavage of protocatechuate.

The $\mathrm{G}+\mathrm{C}$ content is 59.0 to $69.5 \mathrm{~mol} \%$.

The type species is $B$. cepacia (73).

The emended genus Burkholderia contains the following species: $B$. andropogonis comb. nov., B. caryophylli, B. cepacia, $B$. cocovenenans comb. nov., B. gladioli, B. glumae, $B$. mallei, $B$. plantarii, $B$. pseudomallei, $B$. vandii, and $B$. vietnamiensis $\mathrm{sp}$. nov. Descriptions of $B$. caryophylli, $B$. cepacia, $B$. gladioli, $B$. glumae, $B$. mallei, $B$. plantarii, $B$. pseudomallei, and $B$. vandii can be found elsewhere $(2,43,63,73)$. These taxa can be further differentiated on the basis of phenotypic characteristics (Table 2) and genotypic characteristics (Tables 4 and 5).

Description of Burkholderia vietnamiensis sp. nov. Burkholderia vietnamiensis (vi.et'na.mi.en.sis. M.L. adj. vietnamiensis, referring to Vietnam, the country where the rice strains were isolated). Motile cells that are 0.8 to $2 \mu \mathrm{m}$ long and 0.3 to 0.8 $\mu \mathrm{m}$ wide. The colonies on nutrient agar are not pigmented; on King B medium colonies do not produce a fluorescent pigment. Growth occurs on nutrient agar at temperatures between 20 and $41^{\circ} \mathrm{C}$. Not pathogenic for onions. Produces oxidase, catalase, $\beta$-galactosidase, and gelatinase; reduces nitrate to nitrite; and does not produce urease, indole, and arginine dihydrolase. All strains fix atmospheric nitrogen and produce ornibactin siderophores but not pyochelin or cepabactin. Auxanographic characteristics are shown in Table 2 . The characteristics that differentiate this species from $B$. cepacia and $B$. gladioli are shown in Table 6 . The $\mathrm{G}+\mathrm{C}$ contents vary from to
66.9 to $68.1 \mathrm{~mol} \%$. Isolated from rice field soils and from clinical samples. When rRNA from B. cepacia LMG $1222^{\mathrm{T}}$ is used, DNA-rRNA hybrids have $T_{m(e)}$ values similar to the $T_{m(e)}$ values found for members of $B$. cepacia. The species can be recognized on the basis of DNA-DNA homology data.

Type strain TVV75 has been deposited in the collection of the Laboratory of Microbiology in Ghent, Belgium, as strain LMG 10929. The characteristics of the type strain are the same as those described above for the species. The $\mathrm{G}+\mathrm{C}$ content of the type strain is 67.9 mol\%.

Description of Burkholderia cocovenenans (van Damme, Johannes, Cox, and Berends 1960) comb. nov. The description of $B$. cocovenenans is the description given by van Damme et al. (65) and Zhao et al. (74). The type strain is LMG 11626 (= ATCC 33664); the characteristics of the type strain are the same as those of the species. When rRNA from $B$. cepacia LMG $1222^{\mathrm{T}}$ is used, the DNA-rRNA hybrid has a $T_{m(e)}$ of $77.7^{\circ} \mathrm{C}$.

Description of Burkholderia andropogonis (Stapp 1928) comb. nov. Pseudomonas andropogonis (Smith 1911) stapp 1928; Pseudomonas stizolobii (Wolf 1920) Stapp 1935; Aplanobacter stizolobii (Wolf 1920); Pseudomonas Woodsii (Smith 1911) Stevens 1925. In addition to the description given by Palleroni (43), B. andropogonis strains do not grow on L-threonine, L-histidine, and L-tyrosine. More auxanographic characteristics are shown in Table 2. Can be differentiated from other Burkholderia species by its 16S rRNA sequence (38). Pathogenic for sorghum, corn, clover, carnation, and velvet bean. The $\mathrm{G}+\mathrm{C}$ content varies from 59 to $61.3 \mathrm{~mol} \%$. The type strain is LMG 2199 (= NCPPB 934). The description of the type strain is the same as that of the species. The $\mathrm{G}+\mathrm{C}$ content of the type strain is $59 \mathrm{~mol} \%$.

\section{ACKNOWLEDGMENTS}

M.G. and K.K. are indebted to the Nationaal Fonds voor Geneeskundig Onderzoek, Belgium, for research and personnel grants and to bioMérieux for supplying API galleries. T.V.V., P.H., and T.H. are grateful to the European Economic Community for financial support for part of this work.

We are grateful to J. Haurat for technical assistance. We also thank $\mathrm{O}$. Berge for helpful comments and for reading the manuscript.

\section{REFERENCES}

1. Allen, R. N., A. C. Hayward, W. J. Halliday, and J. Fulcher. 1970. Bacteria blight of Vicia sativa; aetiology of the disease and identification of the pathogen. Aust. J. Biol. Sci. 23:597-606.

2. Azegami, K., K. Nishiyama, Y. Watanabe, I. Kadota, A. Ohuchi, and C. Fukazawa. 1987. Pseudomonas plantarii sp. nov., the causal agent of rice seedling blight. Int. J. Syst. Bacteriol. 37:144-152.

3. Ballard, R. W., M. Doudoroff, R. Y. Stanier, and M. Mandel. 1968. Taxonomy of the aerobic pseudomonads: Pseudomonas diminuta and Pseudomonas vesicularis. J. Gen. Microbiol. 53:349-361.

4. Ballard, R. W., N. J. Palleroni, R. Y. Stanier, and M. Mandel. 1970. Taxon omy of the aerobic pseudomonads: Pseudomonas cepacia, $P$. marginata, $P$. aliicola, and $P$. caryophylli. J. Gen. Microbiol. 60:199-214.

5. Bally, R., D. Thomas-Bauzon, T. Heulin, J. Balandreau, C. Richard, and J. De Ley. 1983. Determination of the most frequent $\mathrm{N}_{2}$-fixing bacteria in a rice rhizosphere. Can. J. Microbiol. 29:881-887.

6. Bell, S. C., and J. M. Turner. 1973. Iodinin biosynthesis by a pseudomonad. Biochem. Soc. Trans. 1:751-753.

7. Berge, O., T. Heulin, W. Achouak, C. Richard, R. Bally, and J. Balandreav 1991. Rahnella aquatilis, a nitrogen-fixing enteric bacterium associated with the rhizosphere of wheat and maize. Can. J. Microbiol. 37:195-203.

8. Bowman, J. P., L. I. Sly, and A. C. Hayward. 1988. Pseudomonas mixta, sp. nov., a bacterium from soil with degradative activity on a variety of complex polysaccharides. Syst. Appl. Microbiol. 11:53-59.

9. Bowman, J. P., L. I. Sly, A. C. Hayward, Y. Spiegel, and E. Stackebrandt. 1993. Telluria mixta (Pseudomonas mixta Bowman, Sly, and Hayward 1988) gen. nov., comb. nov., and Telluria chitinolytica sp. nov., soil-dwelling organ- 
isms which actively degrade polysaccharides. Int. J. Syst. Bacteriol. 43:120124.

10. Brenner, D. J., G. R. Fanning, F. J. Skerman, and S. Falkow. 1972. Polynucleotide sequence divergence among strains of Escherichia coli and closely related organisms. J. Bacteriol. 109:953-965.

11. Buddenhagen, I., and A. Kelman. 1964. Biological and physiological aspects of bacterial wilt caused by Pseudomonas solanacearum. Annu. Rev. Phytopathol. 2:203-230.

12. Burbage, D. A., and M. Sasser. 1982. A medium selective for Pseudomonas cepacia. Phytopathol. Abstr. 72:706.

13. Burkholder, W. H. 1957. A bacterial disease of clover and velvet beans. Phytopathology 47:48-50.

14. Burkholder, W. H., and C. E. F. Guterman. 1935. Bacterial leaf spot of carnations. Phytopathology 25:114-120.

15. Caruso, F. L. 1984. Bacterial blight of chickpea incited by Pseudomonas andropogonis. Plant Dis. 68:910-913.

16. Crosa, J. H., D. J. Brenner, and S. Falkow. 1973. Use of a single-strandspecific nuclease for analysis of bacterial and plasmid deoxyribonucleic acid homo- and heteroduplexes. J. Bacteriol. 115:904-911.

17. De Ley, J. 1970. Reexamination of the association between melting point, buoyant density, and chemical base composition of deoxyribonucleic acid. J. Bacteriol. 101:737-754.

18. De Ley, J. 1992. The Proteobacteria: ribosomal RNA cistron similarities and bacterial taxonomy, p. 2109-2140. In A. Balows, H. G. Trüper, M. Dworkin, W. Harder, and K. H. Schleifer (ed.), The prokaryotes, 2nd ed., vol. 2. Springer-Verlag, New York.

19. De Ley, J., and J. De Smedt. 1975. Improvements of the membrane filter method for DNA:rRNA hybridization. Antonie van Leeuwenhoek J. Microbiol. Serol. 41:287-307.

20. De Ley, J., and J. Van Muylem. 1963. Some applications of deoxyribonucleic acid base composition in bacterial taxonomy. Antonie van Leeuwenhoek J. Microbiol. Serol. 29:344-358.

21. De Vos, P., and J. De Ley. 1983. Intra- and intergeneric similarities of Pseudomonas and Xanthomonas ribosomal ribonucleic acid cistrons. Int. J. Syst. Bacteriol. 33:487-509.

22. De Vos, P., M. Goor, M. Gillis, and J. De Ley. 1985. Ribosomal ribonucleic acid cistron similarities of phytopathogenic Pseudomonas species. Int. J. Syst. Bacteriol. 35:169-184.

23. De Vos, P., A. Van Landschoot, P. Segers, R. Tytgat, M. Gillis, M. Bauwens, R. Rossau, M. Goor, B. Pot, K. Kersters, P. Lizzaraga, and J. De Ley. 1989. Genotypic relationships and taxonomic localization of unclassified Pseudomonas and Pseudomonas-like strains by deoxyribonucleic acid-ribosomal ribonucleic acid hybridizations. Int. J. Syst. Bacteriol. 39:35-49.

24. Doudoroff, M., and N. J. Palleroni. 1974. Genus Pseudomonas, p. 217-243. In R. E. Buchanan and N. E. Gibbons (ed.), Bergey's manual of determinative bacteriology, 8th ed. The Williams \& Wilkins Co., Baltimore.

25. Gillis, M., J. Döbereiner, B. Pot, M. Goor, E. Falsen, B. Hoste, B. Reinhold, and K. Kersters. 1991. Taxonomic relationships between [Pseudomonas] rubrisubalbicans, some clinical isolates (EF group 1), Herbaspirillum seropedicae and [Aquaspirillum] autotrophicum. Dev. Plant Soil Sci. 48:293-294.

26. Gonzalez, C., and A. K. Vidaver. 1979. Bacteriocin plasmid and pectolytic diversity in Pseudomonas cepacia of clinical and plant origin. J. Gen. Microbiol. 110:161-170.

27. Goto, M., and M. P. Starr. 1971. A comparative study of Pseudomonas andropogonis, $P$. stizolobii and $P$. alboprecipitans. Ann. Phytopathol. Soc. Jpn. 37:233-241.

28. Grimont, P. A. D., M. Y. Popofi, F. Grimont, C. Coynault, and M. Lemelin. 1980. Reproducibility and correlation study of three deoxyribonucleic acid hybridization procedures. Curr. Microbiol. 4:325-330.

29. Hayward, A. C. 1964. Characteristics of Pseudomonas solanacearum. J. Appl. Bacteriol. 27:265-277.

30. Hayward, A. C. 1972. A bacterial disease of clover in Hawaii. Plant Dis. Rep. 56:446-450.

31. Janse, J. D. 1991. Infra- and intraspecific classification of Pseudomonas solanacearum strains, using whole cell fatty acid analysis. Syst. Appl. Microbiol. 14:335-345.

32. Jenni, B., L. Realini, M. Aragno, and A. U. Tamer. 1988. Taxonomy of non $\mathrm{H}_{2}$-lithotrophic, oxalate-oxidizing bacteria related to Alcaligenes eutrophus. Syst. Appl. Microbiol. 10:123-133.

33. Kersters, K., K. H. Hinz, A. Hertle, P. Segers, A. Lievens, O. Siegmann, and J. De Ley. 1984. Bordetella avium sp. nov., isolated from the respiratory tracts of turkeys and other birds. Int. J. Syst. Bacteriol. 34:56-70.

34. Khambata, S. R., and J. V. Bhat. 1953. Studies on a new oxalate-decomposing bacterium, Pseudomonas oxalaticus. J. Bacteriol. 66:505-507.

35. King, E. O., W. K. Ward, and D. E. Raney. 1954. Two simple media for the demonstration of pyocyanin and fluorescein. J. Lab. Clin. Med. 44:301-307.

36. Kurita, T., and H. Tabei. 1967. On the pathogenic bacterium of bacterial grain rot of rice. Ann. Phytopathol. Soc. Jpn. 33:111.

37. Lapage, S. P., P. H. A. Sneath, E. F. Lessel, V. B. D. Skerman, H. P. R. Seeliger, and W. A. Clark (ed.). 1976. International code of nomenclature of bacteria. 1976 revision. American Society for Microbiology, Washington, D.C.
38. Li, X., M. Dorsch, T. Del Dot, L. I. Sly, E. Stackebrandt, and A. C. Hayward. 1993. Phylogenetic studies of the rRNA group II pseudomonads based on 16S rRNA gene sequences. J. Appl. Bacteriol. 74:324-329.

39. Marmur, J. 1961. A procedure for the isolation of deoxyribonucleic acid from microorganisms. J. Mol. Biol. 3:208-218.

40. Marmur, J., and P. Doty. 1962. Determination of the base composition of deoxyribonucleic acid from its thermal denaturation temperature. J. Mol. Biol. 5:109-118.

41. Meyer, J. M., V. Tran Van, A. Stintzi, H. Stephan, O. Berge, and G. Winkelmann. Unpublished data.

42. Omar, A. M. N., C. Richard, P. Weinhard, and J. Balandreau. 1989. Using the spermosphere model technique to describe the dominant nitrogen-fixing microflora associated with wetland rice in two Egyptian soils. Biol. Fertil. Soils 7:158-163.

43. Palleroni, N. J. 1984. Genus I. Pseudomonas Migula 1894, 237 AL, p. 141-199. In N. R. Krieg and J. G. Holt (ed.), Bergey's manual of systematic bacteriology, vol. 1. The Williams \& Wilkins Co., Baltimore.

44. Palleroni, N. J., and M. Doudoroff. 1971. Phenotypic characterization and deoxyribonucleic acid homologies of Pseudomonas solanacearum. J. Bacteriol. 107:690-696.

45. Palleroni, N. J., and B. Holmes. 1981. Pseudomonas cepacia sp. nov., nom. rev. Int. J. Syst. Bacteriol. 21:479-481.

46. Palleroni, N. J., R. Kunisawa, R. Contopoulou, and M. Doudoroff. 1973. Nucleic acid homologies in the genus Pseudomonas. Int. J. Syst. Bacteriol. 23:333-339.

47. Pot, P., P. Vandamme, and K. Kersters. 1993. Analysis of electrophoretic whole organism protein fingerprints, p. 493-521. In M. Goodfellow and A. G. O'Donnell (ed.), Chemical methods in prokaryotic systematics. John Wiley \& Sons, Chichester, England.

48. Ralston, E., N. J. Palleroni, and M. Doudoroff. 1973. Pseudomonas pickettii, a new species of clinical origin related to Pseudomonas solanacearum. Int. J. Syst. Bacteriol. 23:15-19.

49. Richard, C. 1978. Techniques de recherches d'enzymes utiles au diagnostique de bactéries à Gram négatif. Ann. Biol. Clin. 36:407-424.

50. Roberts, S. J., S. J. Eden-Green, P. Jones, and D. J. Ambler. 1990. Pseudomonas syzygii, sp. nov., the cause of Sumatra disease of cloves. Syst. Appl Microbiol. 13:34-43.

51. Rothwell, A., and A. C. Hayward. 1964. A bacterial disease of bougainvillea. Rhod. J. Agric. Res. 2:97-99.

52. Segers, P., M. Vancanneyt, B. Pot, U. Torck, B. Hoste, D. Dewettinck, E. Falsen, K. Kersters, and P. De Vos. 1994. Classification of Pseudomonas diminuta Leifson and Hugh 1954 and Pseudomonas vesicularis Büsing, Döll, and Freytag 1953 in Brevundimonas gen. nov. as Brevundimonas diminuta comb. nov. and Brevundimonas vesicularis comb. nov., respectively. Int. J. Syst. Bacteriol. 44:499-510.

53. Smith, E. F. 1911. Bacteria in relation to plant diseases. Carnegie Inst. Wash. Publ. 2:1-368.

54. Sneath, P. H. A., and R. R. Sokal. 1973. Numerical taxonomy. The principles and practice of numerical classification. W. H. Freeman and Co., San Francisco.

55. Stackebrandt, E., R. G. E. Murray, and H. G. Trüper. 1988. Proteobacteria classis nov., a name for the phylogenetic taxon that includes the "purple bacteria and their relatives." Int. J. Syst. Bacteriol. 38:321-325.

56. Stanier, R. Y., N. J. Palleroni, and M. Doudoroff. 1966. The aerobic pseudomonads: a taxonomic study. J. Gen. Microbiol. 43:159-271.

57. Stapp, C. 1928. Schizomycetes (Spaltpilze oder bakterien), p. 1-25. In P. Sorauer (ed.), Handbuch der Planzenkrankheiten, 5th ed., vol. 2. Paul Parey, Berlin.

58. Stapp, C. 1935. Contemporary understanding of bacterial plant diseases and their causal organisms. Bot. Rev. 1:405-418.

59. Stead, D. E. 1992. Grouping of plant-pathogenic and some other Pseudomonas spp. by using cellular fatty acid profiles. Int. J. Syst. Bacteriol. 42:281-295.

60. Stephan, H., S. Freund, J. M. Meyer, G. Winkelmann, and G. Jung. 1993. Structure elucidation of the gallium-ornibactin complex by 2D-NMR spectroscopy. Liebigs Ann. Chem. 1993:43-48.

61. Stevens, F. L. 1925. Plant disease fungi. Macmillan Co., New York

62. Tran Van, V. 1989. Isolement et identification des bactéries fixatrices d'azote associées aux racines du riz poussant sur un sol de rizière du Viêt-nam. Mémoire pour Diplome dvétudes approfondies. Université Claude Bernard Lyon I, Lyon France.

63. Urakami, T., C. Ito-Yoshida, H. Araki, T. Kijima, K. I. Suzuki, and K. Komagata. 1994. Transfer of Pseudomonas plantarï and Pseudomonas glumae to Burkholderia as Burkholderia spp. and description of Burkholderia vandii sp. nov. Int. J. Syst. Bacteriol. 44:235-245.

64. Vandamme, P., et al. Unpublished data.

65. van Damme, P. A., A. G. Johannes, H. C. Cox, and W. Berends. 1960. On toxoflavin, the yellow poison of Pseudomonas cocovenenans. Recl. Trav. Chim. Pays-Bas Belg. 79:255-267.

66. Van Landschoot, A, and J. De Ley. 1983. Intra- and intergeneric similarities of the rRNA cistrons of Alteromonas, Marinomonas (gen. nov.) and some other Gram-negative bacteria. J. Gen. Microbiol. 129:3057-3074.

67. Willems, A., J. De Ley, M. Gillis, and K. Kersters. 1991. Comamonadaceae, 
a new family encompassing the acidovorans rRNA complex, including Variovorax paradoxus gen. nov., comb. nov., for Alcaligenes paradoxus (Davis ovorax paradoxus gen. nov., comb. nov.,
1969). Int. J. Syst. Bacteriol. 41:445-450.

68. Willems, A., P. De Vos, M. Gillis, and K. Kersters. 1992. Towards an improved classification of Pseudomonas sp. Soc. Appl. Bacteriol. Tech. Ser. 29:21-43.

69. Wishart, D. 1978. Clustan users manual, 3rd ed. Interuniversity Councils series report 47. Edinburgh University, Edinburgh.

70. Woese, C. R. 1984. Bacterial evolution. Microbiol. Rev. 51:221-271.

71. Woese, C. R., W. G. Weisburg, B. J. Paster, C. M. Hahn, R. S. Tanner, N. R. Krieg, H. P. Koops, H. Harms, and E. Stackebrandt. 1984. The phylogeny of purple bacteria: the beta subdivision. Syst. Appl. Microbiol. 5:327-336.
72. Wolf, F. A. 1920. A bacterial leaf spot of velvet bean. Phytopathology 10:7380 .

73. Yabuuchi, E., Y. Kosako, H. Oyaizu, I. Yano, H. Hotta, Y. Hashimoto, T. Ezaki, and M. Arakawa. 1992. Proposal of Burkholderia gen. nov. and transfer of seven species of the genus Pseudomonas group II to the new genus, with the type species Burkholderia cepacia (Palleroni and Holmes 1981) comb. nov. Microbiol. Immunol. 36:1251-1275.

74. Zhao, N. X., M. S. Ma, Y. P. Zhang, and D. C. Xu. 1990. Comparative description of Pseudomonas cocovenenans (van Damme, Johannes, Cox, and Berends 1960) NCIB $9450^{\mathrm{T}}$ and strains isolated from cases of food poisoning caused by consumption of fermented corn flour in China. Int. J. Syst. Bacteriol. 40:452-455. 